DrAFt VERSION JunE 9, 2021

Typeset using $\mathrm{LAT}_{\mathrm{E}} \mathrm{X}$ default style in AASTeX63

\title{
Molecular Cloud Cores with High Deuterium Fractions: Nobeyama Mapping Survey
}

Ken'ichi Tatematsu, ${ }^{1,2}$ Gwanjeong Kim, ${ }^{1}$ Tie Liu, $, 3,4,5$ Neal J. Evans II, ${ }^{6}$ Hee-Weon Yi,${ }^{7}$ Jeong-Eun Lee, ${ }^{7}$ Yuefang Wu, ${ }^{8}$ Naomi Hirano, ${ }^{9}$ Sheng-Yuan Liu, ${ }^{9}$ Somnath Dutta, ${ }^{9}$ Dipen Sahu, ${ }^{9}$ Patricio Sanhueza, ${ }^{10,2}$ Kee-Tae Kim, ${ }^{11,12}$ Mika Juvela, ${ }^{13}$ L. Viktor Tóth, ${ }^{14}$ Orsolya Fehér, ${ }^{15}$ Jinhua He, ${ }^{16,17,18} \mathrm{~J} . \mathrm{X} . \mathrm{Ge},{ }^{19,18}$ Siyi Feng, ${ }^{20,21,10}$ Minho Choi, ${ }^{4}$ Miju Kang, ${ }^{4}$ Mark A. Thompson, ${ }^{22}$ Gary A. Fuller, ${ }^{23}$ Di Li,${ }^{24}$

Isabelle Ristorcelli, ${ }^{25}$ Ke Wang,${ }^{26,27}$ James Di Francesco,${ }^{28,}{ }^{29}$ David Eden, ${ }^{30}$ Satoshi Ohashi, ${ }^{31}$ Ryo Kandori, ${ }^{32}$

Charlotte Vastel,${ }^{33}$ Tomoya Hirota, ${ }^{10,2}$ Takeshi Sakai, ${ }^{34}$ Xing Lu, ${ }^{10}$ Quang NguyêิN Lu'O'NG, ${ }^{35,4,10,36}$ Hiroko Shinnaga, ${ }^{37}$ Jungha Kim, ${ }^{11,10,38}$ and JCMT Large Program "SCOPE" Collaboration

${ }^{1}$ Nobeyama Radio Observatory, National Astronomical Observatory of Japan, National Institutes of Natural Sciences, 462-2 Nobeyama, Minamimaki, Minamisaku, Nagano 384-1305, Japan

${ }^{2}$ Department of Astronomical Science, The Graduate University for Advanced Studies, SOKENDAI, 2-21-1 Osawa, Mitaka, Tokyo 181-8588, Japan

${ }^{3}$ Shanghai Astronomical Observatory, Chinese Academy of Sciences, 80 Nandan Road, Shanghai 200030, P. R. China

${ }^{4}$ Korea Astronomy and Space Science Institute, Daedeokdaero 776, Yuseong, Daejeon 305-348, South Korea

${ }^{5}$ East Asian Observatory, 660 N. A'ohoku Place, Hilo, HI 96720, USA

${ }^{6}$ Department of Astronomy, The University of Texas at Austin, 2515 Speedway, Stop C1400, Austin, TX 78712-1205, USA

${ }^{7}$ School of Space Research, Kyung Hee University, Seocheon-Dong, Giheung-Gu, Yongin-Si, Gyeonggi-Do, 446-701, Republic of Korea ${ }^{8}$ Department of Astronomy, Peking University, 100871, Beijing, China

${ }^{9}$ Academia Sinica Institute of Astronomy and Astrophysics, 11 F of Astronomy-Mathematics Building, AS/NTU. No.1, Sec. 4, Roosevelt Rd, Taipei 10617, Taiwan, R.O.C.

${ }^{10}$ National Astronomical Observatory of Japan, National Institutes of Natural Sciences, 2-21-1 Osawa, Mitaka, Tokyo 181-8588, Japan

${ }^{11}$ Korea Astronomy and Space Science Institute, Daedeokdaero 776, Yuseong, Daejeon 305-348, Republic of Korea

12 University of Science and Technology, Korea (UST), 217 Gajeong-ro, Yuseong-gu, Daejeon 34113, Republic of Korea

${ }^{13}$ Department of Physics, P.O. Box 64, FI-00014, University of Helsinki, Finland

${ }^{14}$ Department of Astronomy, Eötvös Loránd University, Pázmány Péter sétány 1/A, H-1117 Budapest, Hungary

${ }^{15}$ Institut de Radio Astronomie Millimétrique, 300 rue de la Piscine, Domaine Universitaire 38406 Saint-Martin-d'Heres, France

${ }^{16}$ Yunnan Observatories, Chinese Academy of Sciences, 396 Yangfangwang, Guandu District, Kunming, 650216, China

${ }^{17}$ Chinese Academy of Sciences South America Center for Astronomy, National Astronomical Observatories, CAS, Beijing 100101, China

${ }^{18}$ Departamento de Astronomía, Universidad de Chile, Casilla 36-D, Santiago, Chile

${ }^{19}$ Chinese Academy of Sciences, South America Center for Astrophysics (CASSACA), Camino El Observatorio 1515, Las Condes, Santiago, Chile

${ }^{20}$ Academia Sinica Institute of Astronomy and Astrophysics, No.1, Sec. 4, Roosevelt Rd, Taipei 10617, Taiwan, Republic of China

${ }^{21}$ CAS Key Laboratory of FAST, National Astronomical Observatories, Chinese Academy of Sciences, Beijing 100101, People's Republic of China

${ }^{22}$ Centre for Astrophysics Research, Science $\&$ Technology Research Institute, University of Hertfordshire, Hatfield, AL10 9AB, UK

${ }^{23}$ Jodrell Bank Centre for Astrophysics, School of Physics and Astronomy, University of Manchester, Oxford Road, Manchester, M13 $9 P L, U K$

${ }^{24}$ National Astronomical Observatories, Chinese Academy of Sciences, Beijing, 100012, China

${ }^{25}$ RAP, CNRS (UMR5277), Université Paul Sabatier, 9 avenue du Colonel Roche, BP 44346, F-31028, Toulouse Cedex 4, France

${ }^{26}$ The Kavli Institute for Astronomy and Astrophysics, Peking University, 5 Yiheyuan Road, Haidian District, Beijing 100871, P. R. China

${ }^{27}$ European Southern Observatory, Karl-Schwarzschild-Str. 2 D-85748 Garching bei München, Germany

${ }^{28}$ Herzberg Astronomy \& Astrophysics, National Research Council of Canada, 5071 West Saanich Road, Victoria, BC V9E 2E7, Canada

${ }^{29}$ Department of Physics and Astronomy, University of Victoria, Victoria, BC V8W 2Y2, Canada

${ }^{30}$ Astrophysics Research Institute, Liverpool John Moores University, IC2, Liverpool Science Park, 146 Brownlow Hill, Liverpool L3 5RF, $U K$

${ }^{31}$ The Institute of Physical and Chemical Research (RIKEN), 2-1, Hirosawa, Wako-shi, Saitama 351-0198, Japan

${ }^{32}$ Astrobiology Center of NINS, 2-21-1 Osawa, Mitaka, Tokyo 181-8588, Japan

${ }^{33}$ IRAP, Université de Toulouse, CNRS, CNES, UPS, (Toulouse), France

Corresponding author: Ken'ichi Tatematsu

k.tatematsu@nao.ac.jp 


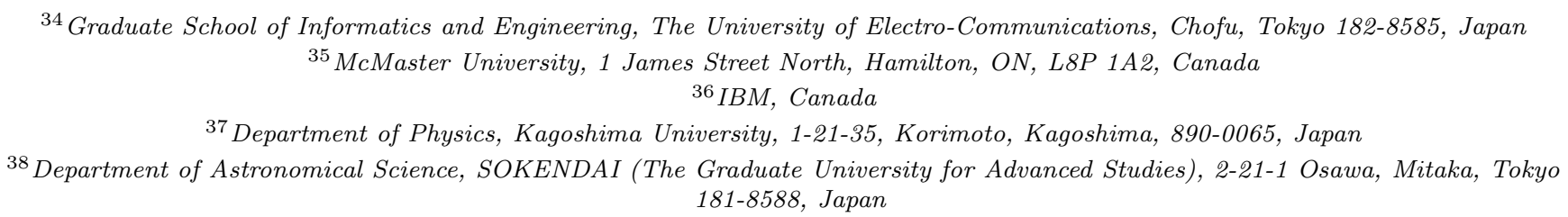

(Received 2021 February 17; Accepted 2021 June 7)

Submitted to ApJS

\begin{abstract}
We present the results of on-the-fly mapping observations of 44 fields containing 107 SCUBA-2 cores in the emission lines of molecules, $\mathrm{N}_{2} \mathrm{H}^{+}, \mathrm{HC}_{3} \mathrm{~N}$, and $\mathrm{CCS}$ at $82-94 \mathrm{GHz}$ using the Nobeyama $45-\mathrm{m}$ telescope. This study aimed at investigating the physical properties of cores that show high deuterium fractions and might be close to the onset of star formation. We found that the distributions of the $\mathrm{N}_{2} \mathrm{H}^{+}$and $\mathrm{HC}_{3} \mathrm{~N}$ line emissions are approximately similar to that of $850-\mu \mathrm{m}$ dust continuum emission, whereas the CCS line emission is often undetected or is distributed in a clumpy structure surrounding the peak position of the $850-\mu \mathrm{m}$ dust continuum emission. Occasionally $(12 \%)$, we observe the CCS emission which is an early-type gas tracer toward the young stellar object, probably due to local high excitation. Evolution toward star formation does not immediately affect nonthermal velocity dispersion.
\end{abstract}

Keywords: ISM: clouds —ISM: molecules —ISM: structure - stars: formation

\title{
1. INTRODUCTION
}

The physical process of the evolution of a molecular cloud core toward the onset of star formation is not clear yet. The timescale toward the onset of star formation (start of the protostar formation), $4 \times 10^{5} \mathrm{yr}$, seems longer than the free-fall time, $10^{5} \mathrm{yr}$ for $N\left(\mathrm{H}_{2}\right)=1 \times 10^{5} \mathrm{~cm}^{-3}$ (Onishi et al. 2002). In addition, as molecular cloud cores are generally close to hydrostatic equilibrium, it is suggested that cores are not (highly) gravitationally unstable. Considering that they are initially stable, star formation involves a mechanism to change stable cores into unstable ones (Nakano 1998). Nakano (1998) suggested that the dissipation of turbulence can be such a mechanism. The dissipation of magnetic fields may work similarly. Gómez et al. (2007) suggested that the mass accretion may cause instability to the core. Star-forming cores are defined as cores associated with young stellar objects including protostars, whereas the other cores are classified as starless cores. Prestellar cores, which are a subset of starless cores, have steep radial density profiles (approximately proportional to $r^{-2}$ in the outer part), suggesting that self-gravity is important for core support (André et al. 1996). Starless cores may evolve to prestellar cores, but not always. Self-gravity becomes more important with increasing steepness of the radial density profile, leading to unstable cores.

Over the past decades, many efforts have been made to study the initial condition of star formation. Recently, an all-sky survey was conducted with the Planck space telescope at (sub)millimeter wavelengths with a large beam $\left(\sim 5^{\prime}\right)$, which provided a catalog of 13200 Planck Galactic Cold Clumps (PGCCs) (Planck Collaboration et al. 2011, 2016). Follow-up observations of the PGCCs, performed with the James Clerk Maxwell Telescope (JCMT) and the SCUBA-2 bolometer at $850 \mu \mathrm{m}$ at higher angular resolution (14"'1), produced catalogs of cores embedded in the PGCCs (Liu et al. 2015, 2018; Yi et al. 2018; Eden et al. 2019). PGCCs have cold dust temperatures $(10-20 \mathrm{~K})$, and SCUBA-2 cores inside them include those with high column densities $\left(\geq 10^{22} \mathrm{~cm}^{-2}\right.$, Kim et al. (2020)). Therefore, SCUBA-2 cores in PGCCs are candidates for prestellar cores in widely different environments such as: nearby dark clouds, giant molecular clouds, and high Galactic latitude clouds. As follow-up observations, we conducted ALMA observations of the SCUBA-2 cores in the dust continuum emission and molecular lines at $1.3 \mathrm{~mm}$, and found substructures in starless cores and star-forming cores in the continuum (Dutta et al. 2020; Sahu et al. 2021). We also performed ammonia observations of the SCUBA cores to derive the rotation, kinetic, and excitation temperatures using the Effelsberg 100 m telescope (Fehér et al. 2020).

Although it is difficult to assess the dynamical evolutionary stages of starless cores, the chemical evolution may provide estimations (Tatematsu et al. 2017). For example, when starless cores evolve toward star formation, the 
Table 1. Numbers of the 107 SCUBA-2 cores in the five regions

\begin{tabular}{lcccc}
\hline \hline \multicolumn{1}{c}{ Region } & No. of fields & No. of cores & No. of starless cores & No. of protostellar cores \\
\hline$\lambda$ Orionis & 1 & 2 & 0 & 2 \\
Orion A & 14 & 36 & 14 & 22 \\
Orion B & 5 & 13 & 5 & 8 \\
Galactic plane & 11 & 30 & 11 & 19 \\
High latitude & 13 & 26 & 4 & 22 \\
\hline Total & 44 & 107 & 34 & 73 \\
\hline Subtotal in the Orion region & 20 & 51 & 19 & 32 \\
\hline
\end{tabular}

deuterium fraction of molecules formed in the gas phase (e.g., DNC/HNC, $\mathrm{N}_{2} \mathrm{D}^{+} / \mathrm{N}_{2} \mathrm{H}^{+}$) increases and attains the maximum at the onset of star formation (Crapsi et al. 2005; Hirota \& Yamamoto 2006; Emprechtinger et al. 2009; Feng et al. 2019). After stellar birth, the deuterium fraction decreases (Fontani et al. 2011; Sakai et al. 2012; Gerner et al. 2015). Moreover, the early-type molecules (e.g., CCS) are abundant in starless cores, whereas the late-type molecules (e.g., $\mathrm{NH}_{3}, \mathrm{~N}_{2} \mathrm{H}^{+}$) are abundant in star-forming cores (Suzuki et al. 1992; Hirahara et al. 1992; Benson et al. 1998; Ohashi et al. 2014, 2016). The ortho-to-para ratio in $\mathrm{H}_{2} \mathrm{D}^{+}$and $\mathrm{D}_{2} \mathrm{H}^{+}$(Pagani et al. 2013; Brünken et al. 2014), the CO depletion (Crapsi et al. 2005; Pagani et al. 2013; Hily-Blant et al. 2020; Feng et al. 2020), and the ${ }^{14} \mathrm{~N} /{ }^{15} \mathrm{~N}$ ratio in the molecule (Redaelli et al. 2020; Hily-Blant et al. 2020) are also used as chemical evolution tracers.

Using the deuterium fraction and early-type/late-type molecules, Tatematsu et al. (2017) proposed the chemical evolution factor (CEF) to evaluate the evolutionary stage of starless cores, herein called CEF1.0. Ge et al. (2020) developed a detailed chemical model for one of the PGCCs that was studied by Tatematsu et al. (2017). Kim et al. (2020) revised the CEF definition (CEF2.0), adding starless cores at distances of $<1 \mathrm{kpc}$, based on a single-pointing survey of 207 SCUBA-2 cores embedded in the PGCCs with the Nobeyama 45-m telescope. The second version of the CEF (CEF2.0) is empirically derived to represent the evolutionary stage of starless cores using the logarithmic deuterium fraction from $\mathrm{N}_{2} \mathrm{D}^{+}$and $\mathrm{DNC}$ as an increasing function. The CEF is defined so that the timing of the onset of star formation corresponds to CEF 0. Here, we explain the difference between CEF1.0 and CEF2.0. CEF1.0 included only nearby dark cloud cores (mostly from Taurus, but also from the Aquila, Serpens and Ophiuchus regions), but CEF2.0 includes also cores in giant molecular clouds (GMCs) in Orion. CEF2.0 only includes the deuterium fraction, which seems a better chemical evolution tracer of the starless core, whereas CEF1.0 also included $N\left(\mathrm{~N}_{2} \mathrm{H}^{+}\right) / N(\mathrm{CCS})$ and $N\left(\mathrm{NH}_{3}\right) / N(\mathrm{CCS})$ as well as the deuterium fraction. Indeed, the chemical evolution sequence of well-known starless cores in Taurus, from L1521B to L1498, and then to L1544 suggested by Shirley et al. (2005) though the SCUBA-2 observations, is consistently described as an increasing function in CEF2.0. Furthermore, CEF2.0 better describes the typical starless molecular cloud core in the Gould Belt by adding Orion GMC cores. It is possible that CEF2.0 may not be relevant for environments outside the Gould Belt, and we need to develop environment-dependent CEFs in the future. Also, note that CEF2.0 is defined using single-dish observations, and the deuterium fractions of structures smaller than $\lesssim 0.01 \mathrm{pc}$ observed with interferometers such as ALMA may be much different from that observed with single-dish telescopes (Sakai et al. 2015). Although we may need further revision, we adopt CEF2.0 as the best description currently available for the Gould Belt. According to Kim et al. (2020), CEF2.0 of the starless SCUBA-2 cores in Orion ranges from -61 to -7 . This CEF2.0 range of $N\left(\mathrm{~N}_{2} \mathrm{D}^{+}\right) / N\left(\mathrm{~N}_{2} \mathrm{H}^{+}\right)$runs from 0.05 to 0.4 , while that of $N(\mathrm{DNC}) / N\left(\mathrm{HN}^{13} \mathrm{C}\right)$ runs from 2 to 8 . If we adopt a ${ }^{12} \mathrm{C} /{ }^{13} \mathrm{C}$ abundance ratio of 43 obtained toward Orion A (Savage et al. 2002), the corresponding $N(\mathrm{DNC}) / N(\mathrm{HNC})$ runs as high as $0.04-0.2$. We take these ranges as typical for starless Orion cores. The Orion SCUBA-2 sources have relatively high deuterium fractions, and are probably at the middle to late stages of the starless core phase.

We report the results of the on-the-fly (OTF) mapping observations of 44 fields including 107 SCUBA-2 cores (Kim et al. 2020) with the Nobeyama $45-\mathrm{m}$ telescope. We selected 65 intense $\mathrm{N}_{2} \mathrm{D}^{+}$cores, 21 high-column-density cores, and their 21 neighboring cores out of 207 SCUBA-2 cores in five regions ( $\lambda$ Orionis, Orion A, B, Galactic plane, and high latitude) observed in our previous single-pointing survey (Kim et al. 2020). The number of cores in each region is summarized in Table 1. The employed distances were specified by Kim et al. (2020). Accurate distances to parent clouds, as available in the literature, were adopted. Otherwise, if no accurate distance was available, the distance of the cloud from the parallax-based distance estimator of the Bar and Spiral Structure Legacy Survey was adopted 
Table 2. Observed Lines

\begin{tabular}{|c|c|c|c|c|c|c|}
\hline Line & $\begin{array}{c}\text { Frequency } \\
\text { GHz }\end{array}$ & Frequency Reference & $\begin{array}{c}\text { Upper Energy Level } E_{u} \\
\mathrm{~K}\end{array}$ & $\begin{array}{l}\text { Critical Density at } 10 \mathrm{~K} \\
\mathrm{~cm}^{-3}\end{array}$ & $\begin{array}{l}\text { HPBW } \\
\prime \prime\end{array}$ & $\begin{array}{c}\text { Velocity Channel Width } \\
\mathrm{km} \mathrm{s}^{-1}\end{array}$ \\
\hline $\operatorname{CCS} J_{N}=7_{6} \rightarrow 6_{5}$ & 81.505208 & Cummins et al. (1986) & 15.3 & $3.1 \times 10^{5}$ & 20 & 0.11 \\
\hline $\operatorname{CCS} J_{N}=8_{7} \rightarrow 7_{6}$ & 93.870107 & Yamamoto et al. (1990) & 19.9 & $4.2 \times 10^{5}$ & 18 & 0.097 \\
\hline $\mathrm{HC}_{3} \mathrm{~N} J=9 \rightarrow 8$ & 81.881462 & Pickett et al. (1998) & 19.7 & $1.1 \times 10^{5}$ & 20 & 0.11 \\
\hline $\mathrm{N}_{2} \mathrm{H}^{+} \quad J=1 \rightarrow 0$ & 93.1737767 & Caselli et al. (1995) & 4.5 & $6.1 \times 10^{4}$ & 18 & 0.098 \\
\hline
\end{tabular}

(Reid et al. 2016), based on the systemic velocity of the line emission and the sky position of the core. The adopted distances are listed in Table A1 in the Appendix. In this study, we focus on the physical properties of Orion cores that are located at similar distances of 350-450 pc (Kounkel et al. 2017; Getman et al. 2019) to draw a reliable comparison between cores by avoiding the serious beam dilution effects as indicated by Kim et al. (2020). For the other cores, we only present the maps without analysis.

This paper is organized as follows. We describe our observations and data analysis in Section 2, present the results of the observations in Section 3, and discuss the physical properties in Section 4. The study is summarized in Section 5 .

\section{OBSERVATIONS}

We conducted mapping observations of 44 fields of $3^{\prime} \times 3^{\prime}$ or larger areas covering 107 SCUBA-2 cores in the $\mathrm{N}_{2} \mathrm{H}^{+}$ $J=1 \rightarrow 0, \mathrm{HC}_{3} \mathrm{~N} J=9 \rightarrow 8, \operatorname{CCS} J_{N}=8_{7} \rightarrow 7_{6}$, and CCS $J_{N}=7_{6} \rightarrow 6_{5}$ lines using the 45-m radio telescope of the Nobeyama Radio Observatory $(\mathrm{NRO})^{1}$ (LP177001; P.I. $=$ K. Tatematsu). The rest frequencies of these four lines, critical densities (Shirley 2015) and other relevant information are summarized in Table 2. The critical densities of the CCS lines were calculated from the Einstein A coefficient and collisional cross section listed in Wolkovitch et al. (1997). Herein, we abbreviate CCS $J_{N}=8_{7} \rightarrow 7_{6}$ and CCS $J_{N}=7_{6} \rightarrow 6_{5}$ to CCS-H and CCS-L, respectively. Observations were performed in the OTF mapping mode (Sawada et al. 2008) from 2017 December to 2019 May. For the receiver frontend, the FOur-beam REceiver System on the 45-m Telescope (FOREST; Minamidani et al. 2016) was used for simultaneous observations of the four lines. The half-power beam width (HPBW) and main-beam efficiency $\eta_{m b}$ at $86 \mathrm{GHz}$ were $19^{\prime \prime} \pm 1^{\prime \prime}$ and $50 \% \pm 4 \%$, respectively. For the receiver backend, the Spectral Analysis Machine for the 45-m telescope (SAM45; Kamazaki et al. 2012) was employed with a channel separation of $30.52 \mathrm{kHz}$, which corresponds to $\sim 0.1 \mathrm{~km} \mathrm{~s}^{-1}$ at $82 \mathrm{GHz}$. The dump time, scan duration, and row spacing are $0.1 \mathrm{sec}, 20 \mathrm{sec}$, and $5^{\prime \prime}$, respectively. The unit map size is either $3^{\prime} \times 3^{\prime}$ or $4^{\prime} \times 4^{\prime}$. When we needed to cover larger fields, we mosaicked unit maps. Then, the scan speed was $11^{\prime \prime} .5 / \mathrm{sec}$ or $14^{\prime \prime} .5 / \mathrm{sec}$ for $3^{\prime} \times 3^{\prime}$ and $4^{\prime} \times 4^{\prime}$ unit maps, respectively. In limited cases, we adopted special rectangular unit maps to cover cores efficiently. We observed unit maps in the R.A. and decl. directions to minimize striping effects. The position switching mode was employed. The typical rms noise level per channel was $0.09 \mathrm{~K}$, and it took approximately $4 \mathrm{hrs}$ to complete a $3^{\prime} \times 3^{\prime}$ unit map. The typical system temperature was $200 \mathrm{~K}$. The telescope pointing calibration was performed at 1.0-1.5 hrs intervals toward $\mathrm{SiO}$ maser sources, which resulted in a pointing accuracy of $\lesssim 5^{\prime \prime}$. The patterns of the telescope main beam and error beam are given in Figure 5 of Minamidani et al. (2016). The maximum height of the error beam is $\lesssim 10 \%$ of the main beam, and the neighboring error beam is located $30^{\prime \prime}-40^{\prime \prime}$ apart from the main-beam. The FWHM size of the neighboring error beam is not very different from that of the main beam.

A total of 44 fields were mapped. Linear baselines were subtracted from the spectral data, and the data were stacked into $5^{\prime \prime}$ spacing pixels with the Bessel-Gauss function on the NOSTAR program (Sawada et al. 2008). The line intensity was expressed in terms of the antenna temperature $T_{\mathrm{A}}^{*}$ corrected for atmospheric extinction using standard chopper wheel calibration.

We also included the SCUBA-2 data (Yi et al. 2018) and our previous single-pointing data toward the SCUBA-2 position (Kim et al. 2020) in the analysis.

\section{RESULTS}

\footnotetext{
${ }^{1}$ Nobeyama Radio Observatory is a branch of the National Astronomical Observatory of Japan, National Institutes of Natural Sciences.
} 


\subsection{Molecular line distribution}

Because the number of maps (44) is large, most maps are presented in the online Figure Set 19, whereas seven representative examples are shown here as Figures 1 to 7 . Table 3 indicates the relationship between the map field and Figure number. These Figures show that, for most of the SCUBA-2 cores, the $\mathrm{N}_{2} \mathrm{H}^{+}$emission distribution is similar to the 850- $\mu \mathrm{m}$ dust continuum distribution. The former is slightly larger than the latter in distribution extent. Difference in the beam sizes for the respective observations may explain part of this difference, but probably not all. The $\mathrm{N}_{2} \mathrm{H}^{+}$emission intensity becomes 'saturated' due to high optical depths for very high densities of $\gtrsim 2 \times 10^{6}$ $\mathrm{cm}^{-3}$, for which the dust continuum will be still sensitive. As a result, the dust continuum traces high-density core centers better, resulting in smaller overall sizes. The $\mathrm{HC}_{3} \mathrm{~N}$ emission shows a distribution similar to the $850-\mu \mathrm{m}$ dust continuum distribution in most of the cores $(\sim 2 / 3)$, again with slightly larger extent, whereas in the remaining cores $(\sim 1 / 3)$, the emission is not detected or surrounds the central region of the $850-\mu \mathrm{m}$ dust continuum emission.

$\mathrm{N}_{2} \mathrm{H}^{+}$can be destroyed by CO evaporated from the dust in warm environments $\left(T_{\text {dust }} \gtrsim 25 \mathrm{~K}\right.$ ) (Jørgensen et al. 2004; Lee et al. 2004; Tatematsu et al. 2014). CCS is basically an early-type molecule, but may have a second abundance enhancement due to the CO depletion before protostar formation ( $\mathrm{Li}$ et al. 2002; Lee et al. 2003). Occasionally, the CCS distribution surrounds the YSO, which reflects different stages of the chemical evolution, or has a intensity peak toward the YSO position due to locally high excitation. These variations will limit the effectiveness of the $\mathrm{N}_{2} \mathrm{H}^{+} / \mathrm{CCS}$ as an chemical evolution tracer in general.

In general, agreement between the SCUBA-2 cores and $\mathrm{N}_{2} \mathrm{H}^{+}$distribution is good. In some cases, the peak positions of the $\mathrm{N}_{2} \mathrm{H}^{+}$emission and the $850-\mu \mathrm{m}$ continuum do not coincide with each other. The star-forming core G208.68-19.20North1 (Figure 4; $N(\mathrm{DNC}) / N\left(\mathrm{HN}^{13} \mathrm{C}\right)=2.6 \pm 1.8, T_{b o l}=38 \pm 13 \mathrm{~K}, T_{k i n}=19.7 \mathrm{~K}$ ) shows such a case. It seems that $\mathrm{N}_{2} \mathrm{H}^{+}$here has been destroyed due to $\mathrm{CO}$ evaporated by local heating due to the YSO. This variation limits the effectiveness of $\mathrm{N}_{2} \mathrm{H}^{+}$as a late-type gas tracer in general. Typically, the spatial variation of $\mathrm{N}_{2} \mathrm{H}^{+}$abundance including chemical evolution will result in the $\mathrm{N}_{2} \mathrm{H}^{+}$distribution different from that of the dust continuum. For example, the southern SCUBA-2 core G211.16-19.33South was detected in the $\mathrm{N}_{2} \mathrm{H}^{+}$emission, but it does not have a prominent peak there. Instead, a prominent $\mathrm{N}_{2} \mathrm{H}^{+}$emission feature is located $1^{\prime}$ east of the SCUBA-2 position. This isolated $\mathrm{N}_{2} \mathrm{H}^{+}$feature is an exceptional example in all the fields in this study, however. It is likely that this isolated feature represents a starless core with less concentration. G211.16-19.33North1, North2 $\left(T_{b o l}=70 \pm 20 \mathrm{~K}\right)$, North4, and North5 $\left(T_{b o l}=112 \pm 16 \mathrm{~K}\right)$, which were detected in both the continuum and $\mathrm{N}_{2} \mathrm{H}^{+}$, are star-forming cores. Note that the starless core G211.16-19.33North3 detected in the continuum and $\mathrm{N}_{2} \mathrm{H}^{+}$shows infall motions in the ALMA ACA observations (Tatematsu et al. 2020).

The CCS emission shows a clumpy distribution, which is frequently very different from the $850-\mu \mathrm{m}$ dust continuum distribution, or is simply not detected in most of the cores. The spatial distribution of the line emission is variable from core to core. For the star-forming core G204.4-11.3A2East (Fig. 1; $N\left(\mathrm{~N}_{2} \mathrm{D}^{+}\right) / N\left(\mathrm{~N}_{2} \mathrm{H}^{+}\right)=0.27 \pm 0.07$ ), the $\mathrm{N}_{2} \mathrm{H}^{+}$ and $\mathrm{HC}_{3} \mathrm{~N}$ lines show a distribution similar to the $850-\mu \mathrm{m}$ distribution, whereas the CCS-H and -L lines show clumpy distributions, which are very different from the $850-\mu \mathrm{m}$ distribution. Furthermore, the CCS-L emission appears to surround the dust continuum core, which can be explained in terms of the chemical evolution; the gas in the vicinity of the YSO is more evolved. All four lines are detected in the star-forming core G206.93-16.61West3 (Fig. 3) and show distributions similar to the $850-\mu \mathrm{m}$ distribution. It is possible that local warmer temperatures around the YSO provide high excitation for both the CCS-H and -L emission lines to be detected. The upper energy levels of the CCS-H and CCS-L are 19.9 K and 15.3 K, respectively. Toward the starless cores G206.93-16.61West4 and West5 (Figure 3), we did not detect the CCS emission but only the $\mathrm{N}_{2} \mathrm{H}^{+}$emission. Their CEF2.0 values are $-27 \pm 14$ and $-34 \pm 3$, respectively, and they are thought to be evolved starless cores. Furthermore, it is possible that the CCS depletion occurs in cold starless cores. The star-forming core G212.10-19.15South (Figure $7 ; N\left(\mathrm{~N}_{2} \mathrm{D}^{+}\right) / N\left(\mathrm{~N}_{2} \mathrm{H}^{+}\right)=$ $0.39 \pm 0.06)$ with a YSO with low bolometric temperature $\left(T_{b o l}=43 \pm 12 \mathrm{~K}\right)$ does not show any CCS emission. Another YSO with a low bolometric temperature $\left(T_{b o l}=49 \pm 21 \mathrm{~K}\right)$ associated with G211.47-19.27South (Figure 6) does show CCS-H emission, whereas CCS-L emission surrounds it as if it were avoiding the YSO. The YSO associated with G211.16-19.27North1 (Figure 5) accompanies the CCS-L emission, but does not show intense CCS-H emission. We detected both CCS-H and -L emission toward the YSO in G206.93-16.61West3 (Figure 3; $N\left(\mathrm{~N}_{2} \mathrm{D}^{+}\right) / N\left(\mathrm{~N}_{2} \mathrm{H}^{+}\right)=$ $0.04 \pm 0.02, N(\mathrm{DNC}) / N\left(\mathrm{HN}^{13} \mathrm{C}\right)=1.7 \pm 1.2$ ). The star-forming core G212.10-19.15North2 (Figure 7) accompanying a YSO having $T_{b o l}=114 \pm 10 \mathrm{~K}$ is associated with CCS-L emission, and also with very weak CCS-H emission. $12 \%$ of the Orion starless cores show local CCS peak emission toward YSOs in either CCS-H or -L. The CCL-H emission 
has a higher upper energy level (Table 2), but we do not see a very clear tendency that it is more concentrated on the YSO position than the CCS-L emission.

The line detection statistics, line profiles, and column density ratios toward the SCUBA-2 positions are fully presented in our single-pointing paper (Kim et al. 2020). We expect that the telescope error beam will not seriously affect the observed molecular distribution. Because the beam efficiency is high, the line emission distribution is very clumpy with sizes of $\sim 1^{\prime}$, and the apparent area filling factor of the emission is not very large.

Table 3. Field list

\begin{tabular}{|c|c|}
\hline Field & Figure Number \\
\hline G192 & 19.1 \\
\hline G203 & 19.2 \\
\hline G204 & 1 \\
\hline G206.12 & 2 \\
\hline G206.21 & 19.3 \\
\hline G206.93 & 3 \\
\hline G207 & 19.4 \\
\hline G208.68 & 4 \\
\hline G208.89 & 19.5 \\
\hline G209.05 & 19.6 \\
\hline G209.29North & 19.7 \\
\hline G209.29South & 19.8 \\
\hline G209.77 & 19.9 \\
\hline G209.94North & 19.10 \\
\hline G209.94South & 19.11 \\
\hline G210 & 19.12 \\
\hline G211.16 & 5 \\
\hline G211.47 & 6 \\
\hline G211.72 & 19.13 \\
\hline G212 & 7 \\
\hline G159 & 19.14 \\
\hline G171 & 19.15 \\
\hline G172 & 19.16 \\
\hline G173 & 19.17 \\
\hline G178 & 19.18 \\
\hline G006 & 19.19 \\
\hline G001 & 19.20 \\
\hline G17 & 19.21 \\
\hline G14 & 19.22 \\
\hline G16.96 & 19.23 \\
\hline G16.36 & 19.24 \\
\hline G24 & 19.25 \\
\hline G33 & 19.26 \\
\hline G35 & 19.27 \\
\hline G34 & 19.28 \\
\hline G57 & 19.29 \\
\hline G69 & 19.30 \\
\hline G74 & 19.31 \\
\hline G82 & 19.32 \\
\hline G91 & 19.33 \\
\hline G92 & 19.34 \\
\hline G105 & 19.35 \\
\hline G93 & 19.36 \\
\hline G107 & 19.37 \\
\hline
\end{tabular}

3.2. Line emission distribution in representative cores 


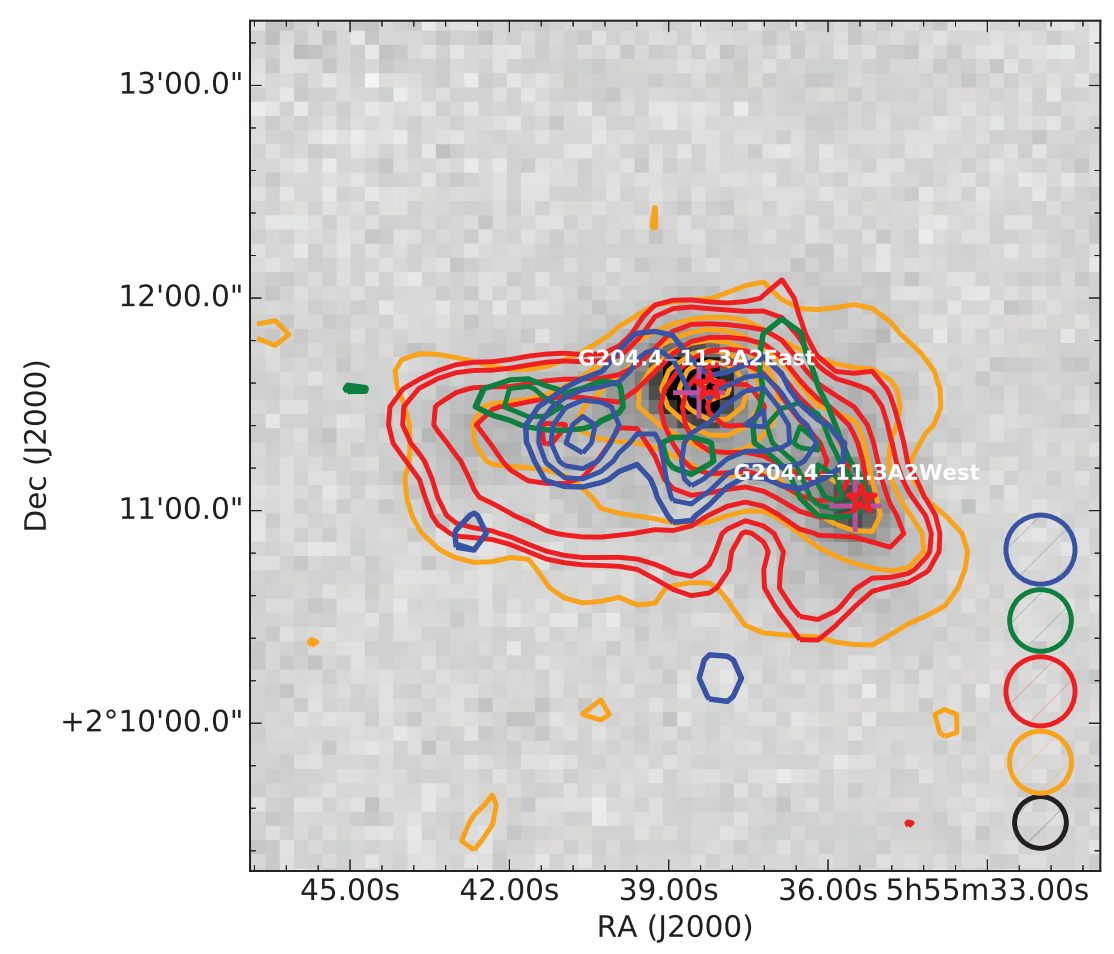

Figure 1. NRO FOREST maps of $\mathrm{N}_{2} \mathrm{H}^{+}$(orange), $\mathrm{HC}_{3} \mathrm{~N}$ (red), CCS-H (green), and CCS-L (blue) integrated intensities overlaid on JCMT SCUBA-2 850- $\mu$ m continuum emission, in gray scale, for field G204.4. The contours are drawn at 5\%, 20\%, $35 \%, 50 \%, 65 \%, 80 \%$, and $95 \%$ of the peak value above $3 \sigma$, and also at $3 \sigma$. The respective peak and $\sigma$ values of integrated intensity are listed in Table A3. The circles represent the beam sizes with corresponding colors. The black circle, however, corresponds to the SCUBA-2 beam size. The cross and star symbols represent a SCUBA-2 core and a protostar, respectively.

We investigated two extreme cases in detail: one case G212 where all the four lines have the same peaks, and another case G206.12 where the distributions of the continuum, $\mathrm{N}_{2} \mathrm{H}^{+}$, and $\mathrm{HC}_{3} \mathrm{~N}$ emission are different from that of the CCS emission.

First, we considered field G212 (Figure 7). We detect intense emission in CCS-H and -L toward the YSO associated with G212.10-19.15North3 $\left(N\left(\mathrm{~N}_{2} \mathrm{D}^{+}\right) / N\left(\mathrm{~N}_{2} \mathrm{H}^{+}\right)=0.06 \pm 0.03\right.$ and $\left.N(\mathrm{DNC}) / N\left(\mathrm{HN}^{13} \mathrm{C}\right)=2.8 \pm 2.0\right)$. CCS is usually regarded as an early-type gas tracer, but the CCS emission peak coincides with the YSO on this core. In the SCUBA-2 peak G212.10-19.15North1 $\left(N\left(\mathrm{~N}_{2} \mathrm{D}^{+}\right) / N\left(\mathrm{~N}_{2} \mathrm{H}^{+}\right)=0.35 \pm 0.07\right)$, the four molecular lines are all distributed similarly, but their peaks are displaced by $\sim 40^{\prime \prime}$ or 0.08 pc from the SCUBA-2 peak. It is possible that the YSO is destroying the core. Another possibility is that the YSO has moved from its birth site due to proper motion (Tatematsu et al. 2014), although we do not know the accurate age of the YSO or the vector of its proper motion with respect to the sky plane.

Next, we investigated the radial intensity distribution field G206.12 (Figure 2), which contains only one SCUBA-2 core $\mathrm{G} 206.12-15.76\left(N(\mathrm{DNC}) / N\left(\mathrm{HN}^{13} \mathrm{C}\right)<4.8\right)$ but was detected with all four lines. This core contains a YSO $\left(T_{b o l}=35 \pm 9 \mathrm{~K}\right)$ near the SCUBA-2 position $\left(2^{\prime \prime} \cdot 9 \mathrm{SSW}\right)$. We binned the continuum and integrated-intensity line maps to $20^{\prime \prime}$ pixels, which is close to the NRO telescope beam size. Figure 8 compares the intensity normalized for the maximum pixel value in the SCUBA-2 $850-\mu \mathrm{m}$ continuum and the line against offset from the map center. The values at the same offset from the map center (SCUBA-2 position) were averaged. For 850- $\mu$ m continuum, $\mathrm{N}_{2} \mathrm{H}^{+}$and $\mathrm{HC}_{3} \mathrm{~N}$ have their maxima at the core center, which is almost identical to the YSO position. The $850-\mu \mathrm{m}$ continuum, however, decreases more sharply than those of $\mathrm{N}_{2} \mathrm{H}^{+}$and $\mathrm{HC}_{3} \mathrm{~N}$. Differences in the telescope beam radius $\left(7^{\prime \prime}\right.$ and $9^{\prime \prime}-10^{\prime \prime}$ for the $850-\mu \mathrm{m}$ continuum and the lines, respectively) may affect this difference, but also the molecular lines will be 'saturated' with high optical depths for very high densities as explained in §3. CCS-H and CCS-L seem to have a 


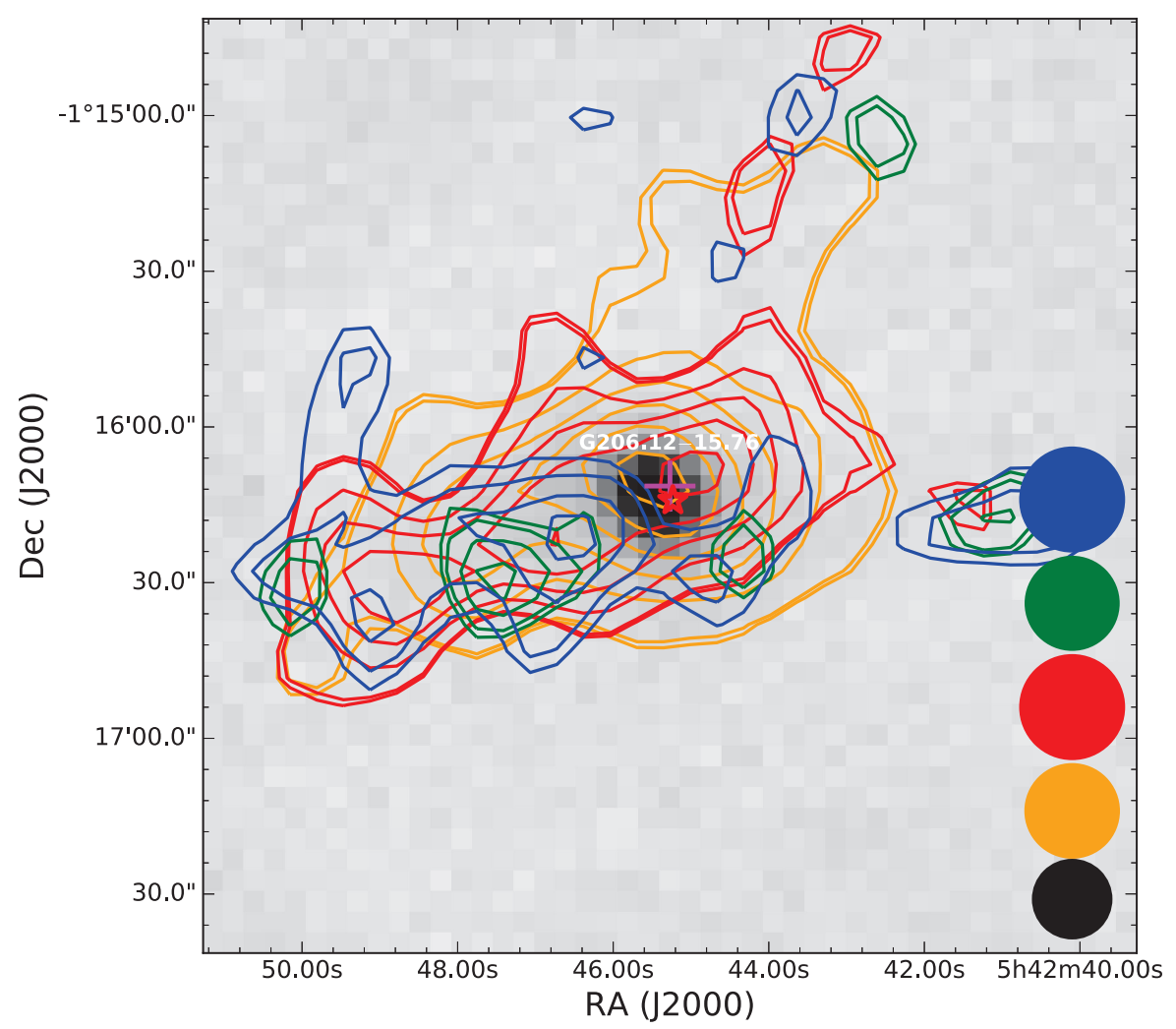

Figure 2. Same as Figure 1 but for field G206.12.

depression toward the core center. It is known that CCS also exists in evolved molecular gas as a secondary late-stage peak due to CO depletion. The observed emission could represent such gas. It seems that the gas near the core center is more chemically evolved so that the CCS abundance is reduced.

\subsection{Physical properties of the SCUBA-2 core}

We investigated the physical properties of the SCUBA-2 cores using the $\mathrm{N}_{2} \mathrm{H}^{+}$data toward the SCUBA-2 position (Kim et al. 2020). We adopted the hyperfine spectral fitting result for the $\mathrm{N}_{2} \mathrm{H}^{+}$spectrum. We neglected cores with two $\mathrm{N}_{2} \mathrm{H}^{+}$velocity components, as it was difficult to identify the component that corresponded to the SCUBA-2 emission. The nonthermal and total velocity dispersions, $\sigma_{\mathrm{nt}}$ and $\sigma_{\text {tot }}$, are defined as Fuller \& Myers (1992):

$$
\sigma_{\mathrm{nt}}=\sqrt{\frac{\Delta v_{\mathrm{obs}}^{2}}{8 \ln 2}-\frac{k_{\mathrm{B}} T_{\mathrm{kin}}}{\mu_{\mathrm{obs}} m_{\mathrm{H}}}}
$$

and

$$
\sigma_{\mathrm{tot}}=\sqrt{\frac{\Delta v_{\mathrm{obs}}^{2}}{8 \ln 2}+\frac{k_{\mathrm{B}} T_{\mathrm{kin}}}{m_{\mathrm{H}}}\left(\frac{1}{\mu}-\frac{1}{\mu_{\mathrm{obs}}}\right)}
$$






Figure 3. Same as Figure 1 but for field G206.93.

respectively, where $\Delta v_{\text {obs }}$ is the FWHM linewidth, $k_{\mathrm{B}}$ is the Boltzmann constant, and $T_{\text {kin }}$ is the kinetic temperature. We assume that the kinetic temperature is equal to the dust temperature. $\mu_{\text {obs }}$ is the molecular weight of the observed molecule in units of the hydrogen mass $m_{\mathrm{H}}$. The mean molecular weight $\mu$ per particle in units of $m_{\mathrm{H}}$ is set to 2.33.

In this study, we adopted the HWHM radius $R$ (dust) and mass $M$ (dust) of the core from the SCUBA-2 results of Yi et al. (2018) to avoid uncertainties of the $\mathrm{N}_{2} \mathrm{H}^{+}$abundance. Yi et al. (2018) expressed the core size $R$ in terms of the FWHM diameter, but we express $R$ in terms of the HWHM radius here.

The virial mass $M_{\text {vir }}$ of the uniform-density sphere is derived using the following formula (McKee \& Zweibel 1992):

$$
M_{\mathrm{vir}}=\frac{5 R \sigma_{\mathrm{tot}}^{2}}{G}
$$

where $G$ is the gravitational constant. The virial parameter $\alpha_{\text {vir }}$ is estimated by dividing the virial mass by the SCUBA-2 core mass,

$$
\alpha_{v i r}=\frac{M_{v i r}}{M(\text { dust })} .
$$




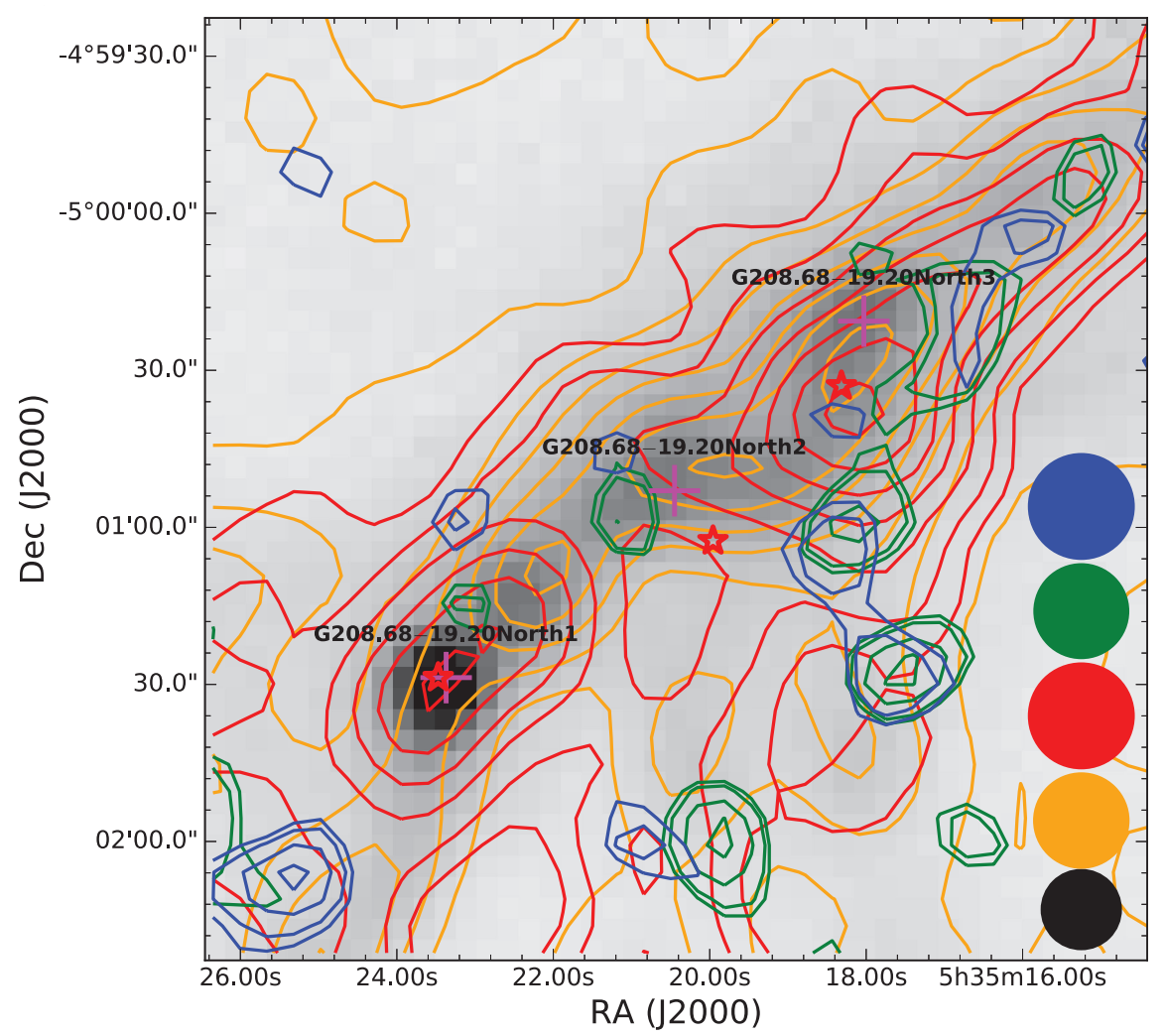

Figure 4. Same as Figure 1 but for field G208.68.

Table 4 lists the velocity dispersions, radii, masses, virial masses, and virial parameters for the SCUBA-2 cores. The virial parameter is as large as $\sim 5$. Figure 9 plots the virial parameter as a function of SCUBA-2 core mass. We adopted the uncertainty in the core radius to be $8 \%$ from the uncertainty in the distance adopted by Yi et al. (2018), and derived the uncertainty in the virial parameter assuming the propagation of random errors. From this sample, we obtained the least-squares fit to be $\alpha_{v i r} \propto M$ (dust) ${ }^{-0.7}$, which can be well described in terms of a pressure-confined core with a form $\alpha_{v i r} \propto M^{-2 / 3}$ (Bertoldi \& McKee 1992). For the core located near $\alpha_{v i r} \sim$ unity, self-gravity is likely to be dominant if we assume that it is in hydrostatic equilibrium. The core with an $\alpha_{v i r}$ value that is considerably larger than unity probably needs an appropriate external pressure to bind it if it is not a transient object. Similar results showing large virial parameters are obtained by Kirk et al. (2017) and Kerr et al. (2019) in Orion and in other Gould Belt clouds, respectively. Note that core G211.47-19.27South has a very small virial parameter, $\alpha_{v i r}=0.1$, and was cataloged by Yi et al. (2018) with an exceptionally small size, which is one order of magnitude smaller than the other cores. Its deconvolved size is smaller than the telescope beam. We define a subcategory of $M$ (dust) $>2 M_{\odot}$, which seems closer to virial equilibrium. We estimate $\alpha_{v i r}$ to be $5.9 \pm 4.6$ and $2.5 \pm 1.2$ for all the cores and for the subcategory $M$ (dust) $>2 M_{\odot}$, respectively. It should be noted that the virial parameter for the structure embedded in the larger structure can be affected by tidal forces (Mao et al. 2012) In addition, the virial parameter can be affected by internal motions (Ayushi Singh, Christopher Matzner, and the Green Bank Ammonia Collaboration, private communication). 


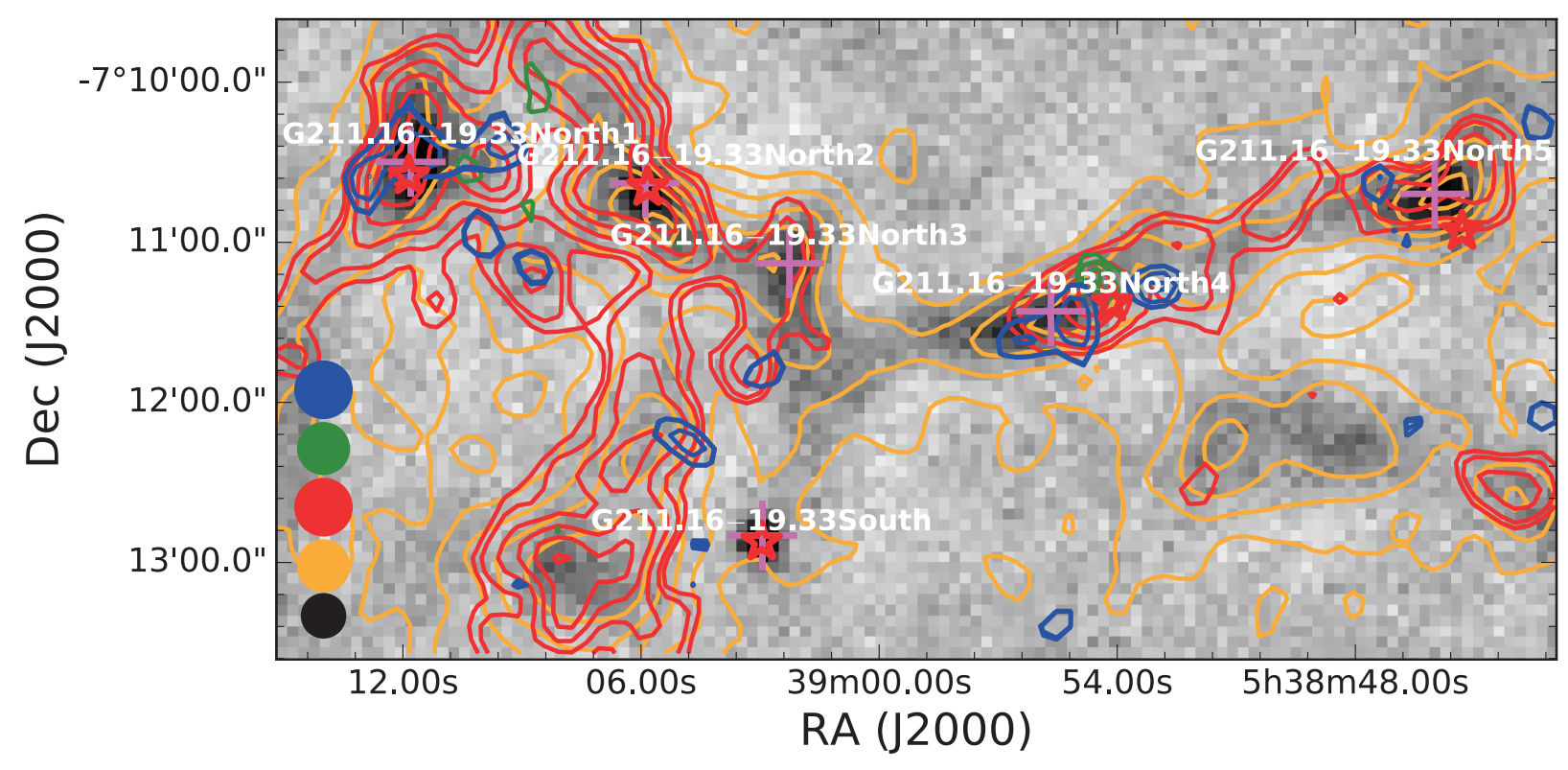

Figure 5. Same as Figure 1 but for field G211.16. 


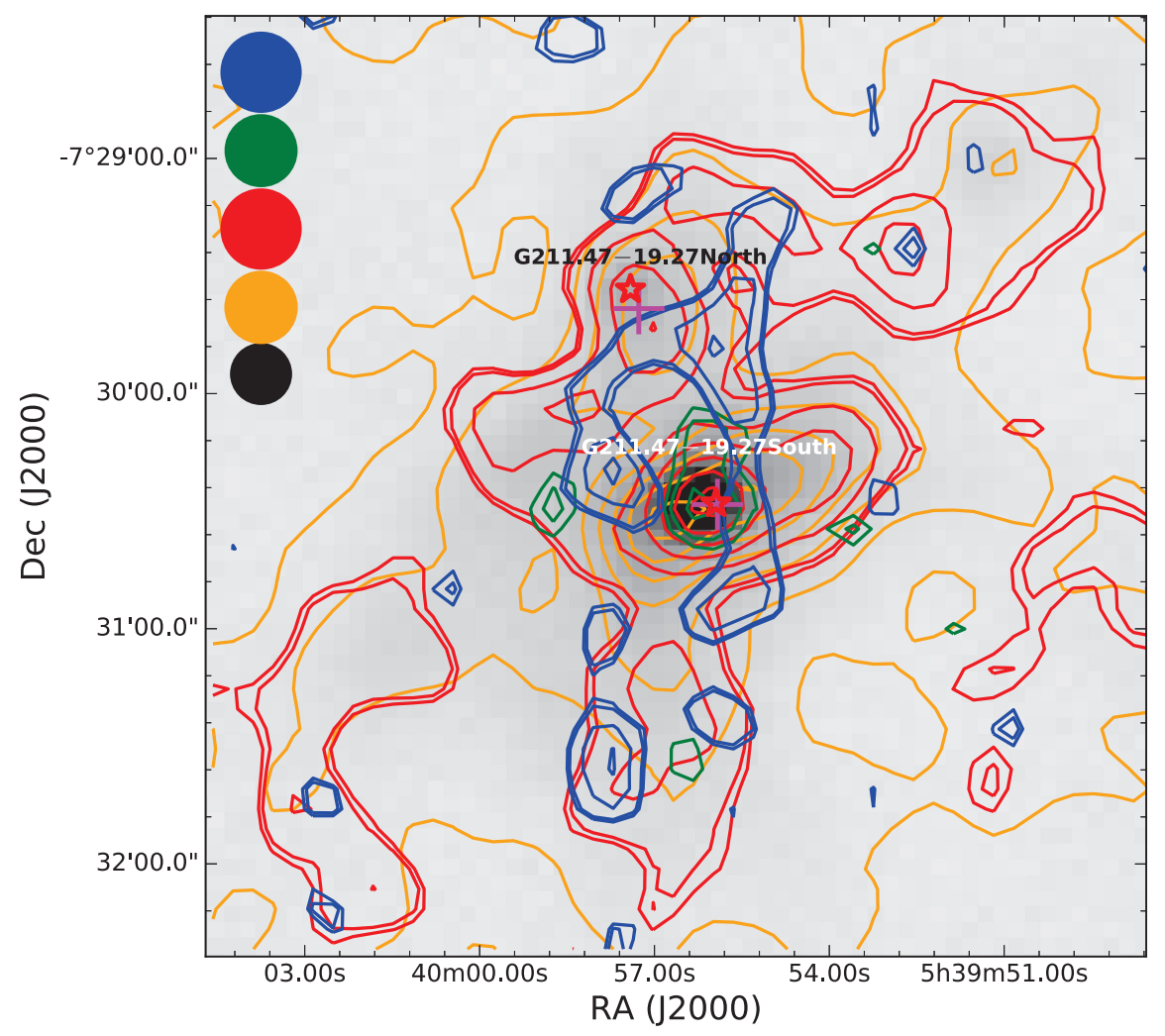

Figure 6. Same as Figure 1 but for field G211.47. 


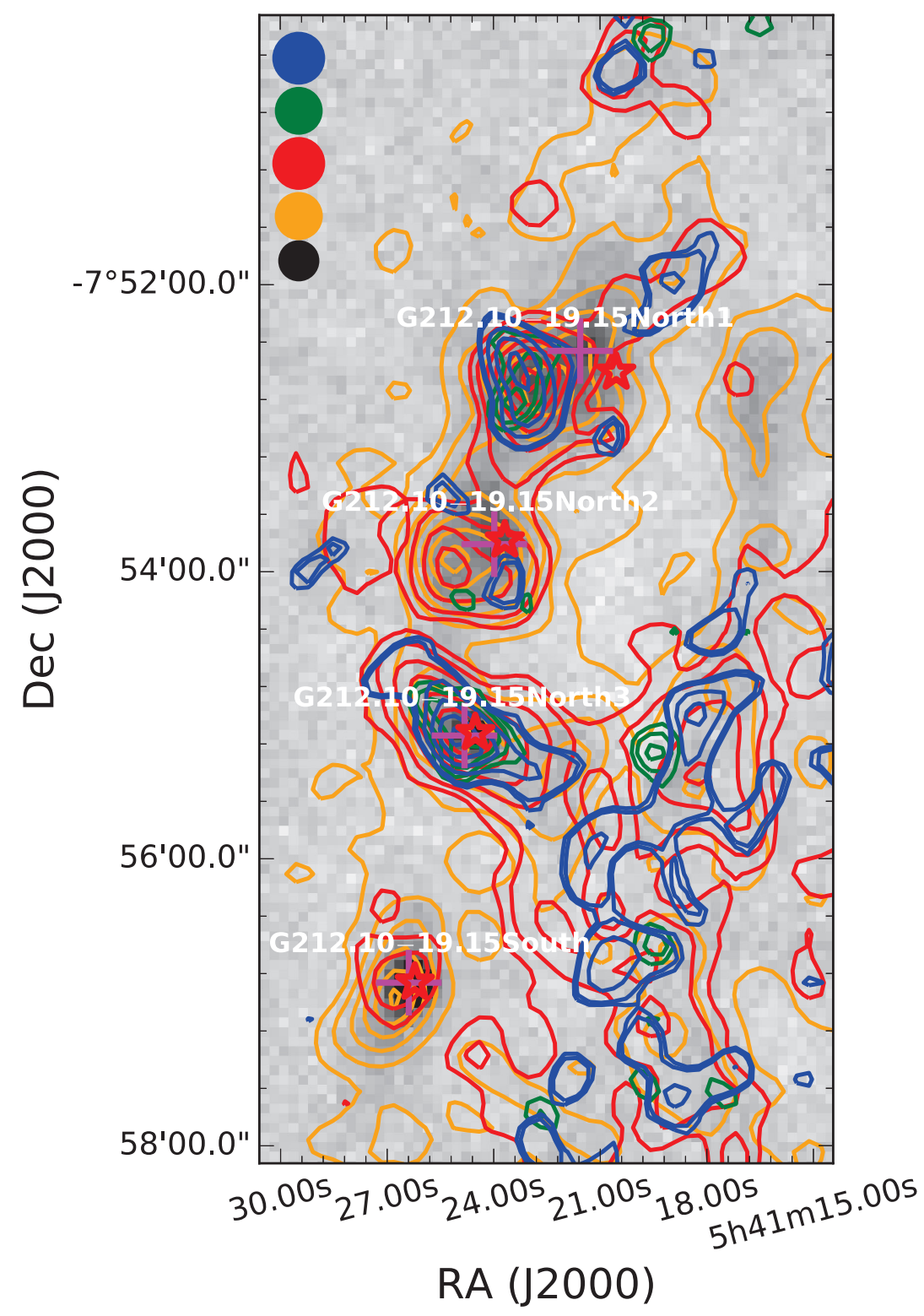

Figure 7. Same as Figure 1 but for field G212. 


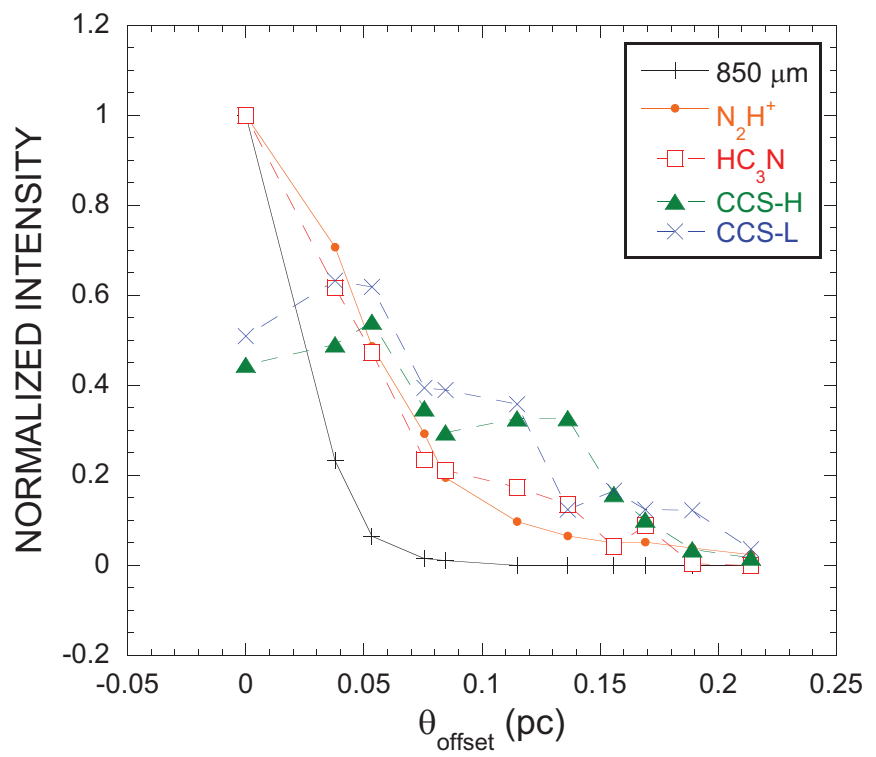

Figure 8. Radial distribution of the continuum and line intensity normalized to the maximum value, toward the star-forming core G206.12-15.76 (Figure 2). 


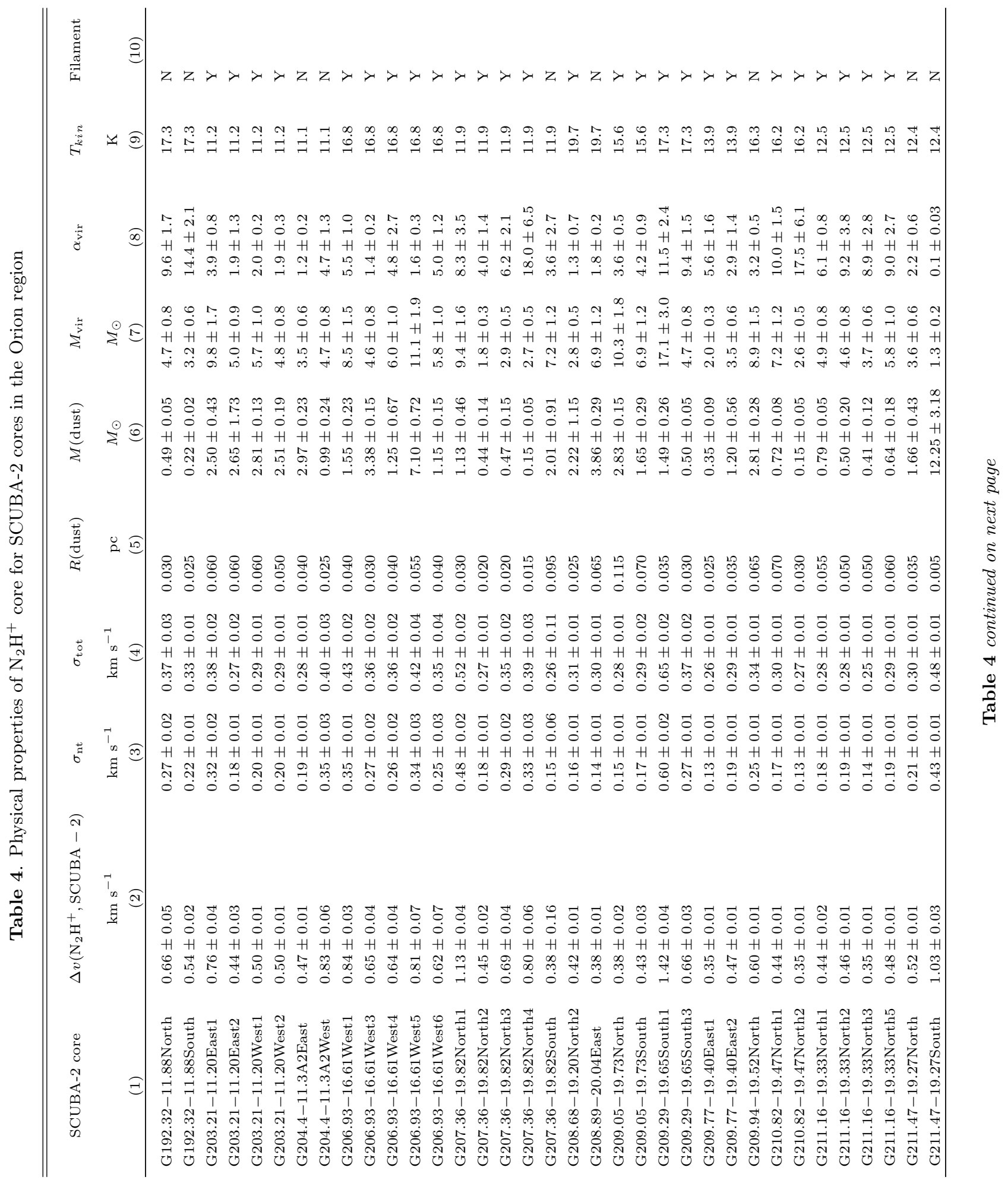




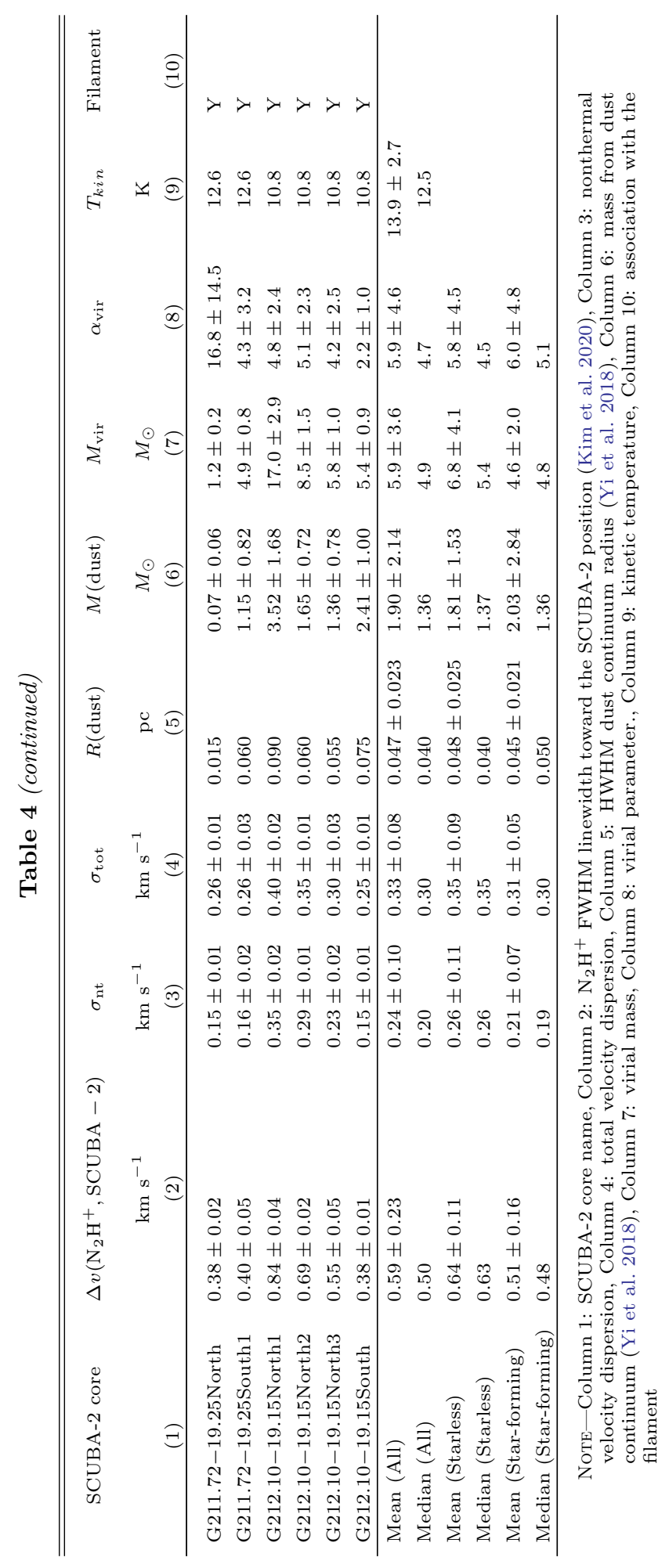




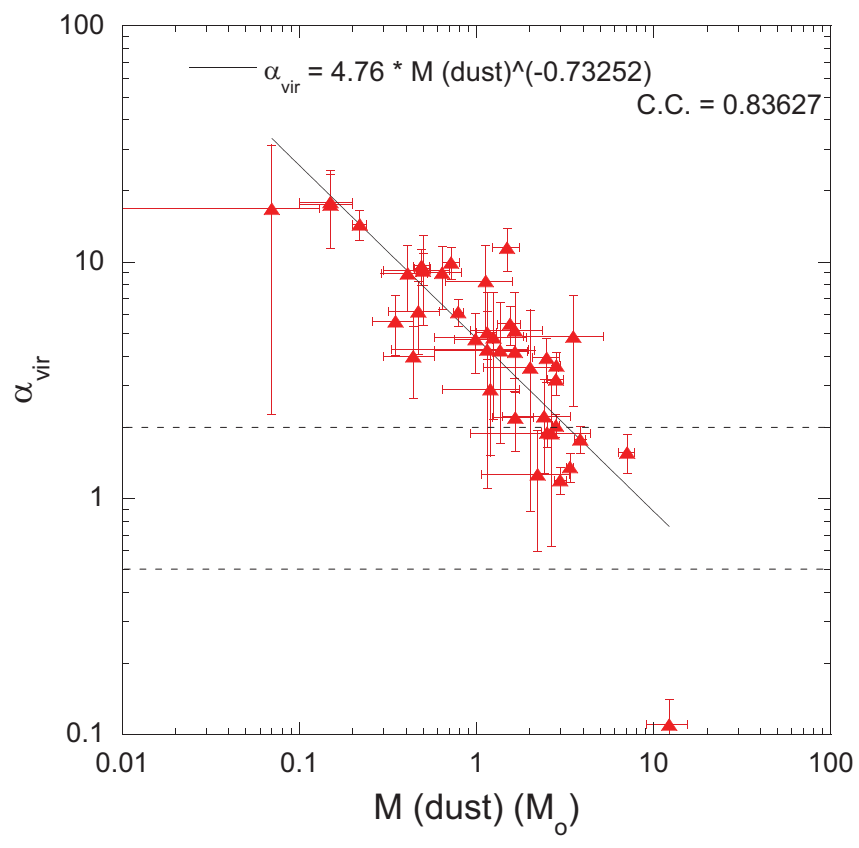

Figure 9. Virial parameter $\alpha_{v i r}$ vs. $M$ (dust). The straight line was computed using a least-squares program. C.C represents the correlation coefficient. The horizontal dotted lines represents $\alpha_{v i r}=0.5$ and 2 .

\subsection{Line emission distribution of the Orion core}

We statistically compared the emission distributions of the four lines on the basis of their integrated-intensity maps toward Orion cores. We selected the emission feature that was approximately coincident with the SCUBA-2 850- $\mu \mathrm{m}$ dust continuum emission through visual inspection and fitted a two-dimensional Gaussian to it. We identified the most prominent feature that was the nearest to the SCUBA-2 position. Although this description may sound somewhat imprecise, the identification is practically straightforward.

The individual core data are listed in Tables A4 and A5 in the Appendix. Table 5 summarizes statistics for the offset $\theta_{\text {offset }}$ of the Gaussian-fit center from the SCUBA-2 position and the beam deconvolved radius $R$ of the fit. Note that the offset of CCS-L is twice as large as those of $\mathrm{N}_{2} \mathrm{H}^{+}$and CCS-H. Therefore, CCS-L emission is in general less correlated with the SCUBA-2 emission. We did not observe large differences in radius between the lines.

We also illustrate the offset and emission radius by histogram. Figure 10 provides a comparison of the offset of the line emission fitted ellipse center from the SCUBA-2 position for the Orion cores. The offsets in $\mathrm{N}_{2} \mathrm{H}^{+}$and $\mathrm{HC}_{3} \mathrm{~N}$ are similarly distributed, having sharp peaks at $\theta_{\text {offset }}=0-0.02 \mathrm{pc}$, whereas those in CCS-H and CCS-L appear to be less peaked. The NRO telescope beam radius is 0.018 pc. Figure 11 shows a comparison of the deconvolved radius values of the line emission feature for the Orion cores, in which no significant differences among the lines are observed.

\subsection{Comparison between the starless and star-forming cores in the Orion region}

At the bottom of Table 4, we list parameter statistics for all the cores together, and starless and star-forming cores separately. The starless and star-forming cores have very similar radii, suggesting no evolutionary change in the core radius is detected at 0.027 -pc linear resolution. Furthermore, their nonthermal velocity dispersions, masses, and virial masses are not appreciably different. Therefore, no evidence for the dissipation of turbulence is identified 
Table 5. Line emission distribution summary in the Orion region

\begin{tabular}{lccccccccc}
\hline \hline SCUBA-2 core & \multicolumn{2}{c}{$\mathrm{N}_{2} \mathrm{H}^{+}$} & \multicolumn{2}{c}{$\mathrm{HC}_{3} \mathrm{~N}$} & \multicolumn{2}{c}{ CCS-H } & \multicolumn{2}{c}{ CCS-L } \\
\cline { 2 - 10 } & $\theta_{\text {offset }}$ & $R$ & $\theta_{\text {offset }}$ & $R$ & $\theta_{\text {offset }}$ & $R$ & $\theta_{\text {offset }}$ & $R$ \\
& $\mathrm{pc}$ & $\mathrm{pc}$ & $\mathrm{pc}$ & $\mathrm{pc}$ & $\mathrm{pc}$ & $\mathrm{pc}$ & $\mathrm{pc}$ & $\mathrm{pc}$ \\
& $(2)$ & $(3)$ & $(4)$ & $(5)$ & $(6)$ & $(7)$ & $(8)$ & $(9)$ \\
\hline \multirow{2}{*}{$(1)$} & 0.027 & 0.073 & 0.031 & 0.065 & 0.021 & 0.062 & 0.045 & 0.081 \\
mean & 0.026 & 0.027 & 0.036 & 0.026 & 0.022 & 0.027 & 0.041 & 0.040 \\
\hline
\end{tabular}

Note-Column 1: Calculation, Column 2: offset of the $\mathrm{N}_{2} \mathrm{H}^{+}$emission from the SCUBA-2 position in pc, Column 3: deconvolved radius of the $\mathrm{N}_{2} \mathrm{H}^{+}$emission in pc, Column 4: offset of the $\mathrm{HC}_{3} \mathrm{~N}$ emission from the SCUBA-2 position in pc, Column 5: deconvolved radius of $\mathrm{HC}_{3} \mathrm{~N}$ emission in pc, Column 6: offset of the CCS-H emission from the SCUBA-2 position in pc, Column 7: deconvolved radius of the CCS-H emission in pc, Column 8: offset of the CCS-L emission from the SCUBA-2 position in pc, and Column 9: deconvolved radius of CCS-L emission in pc.

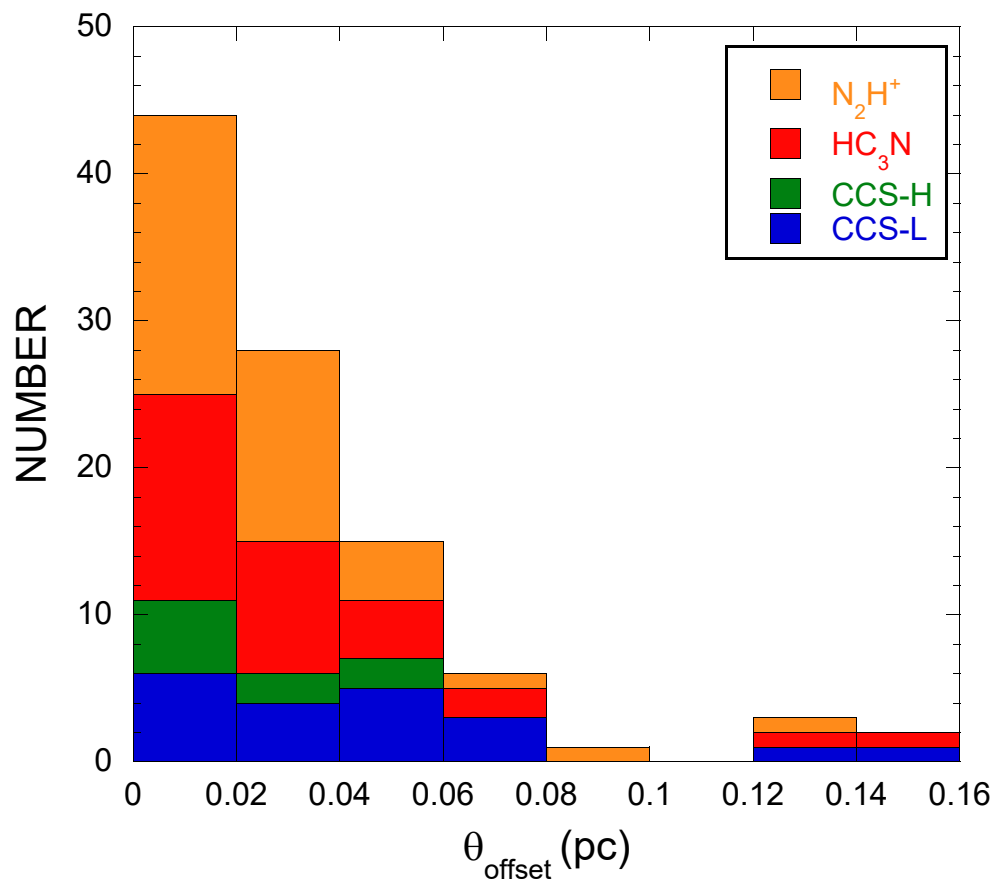

Figure 10. Histogram of the line emission offset from the SCUBA-2 position. Statistics are listed in Table 5. 


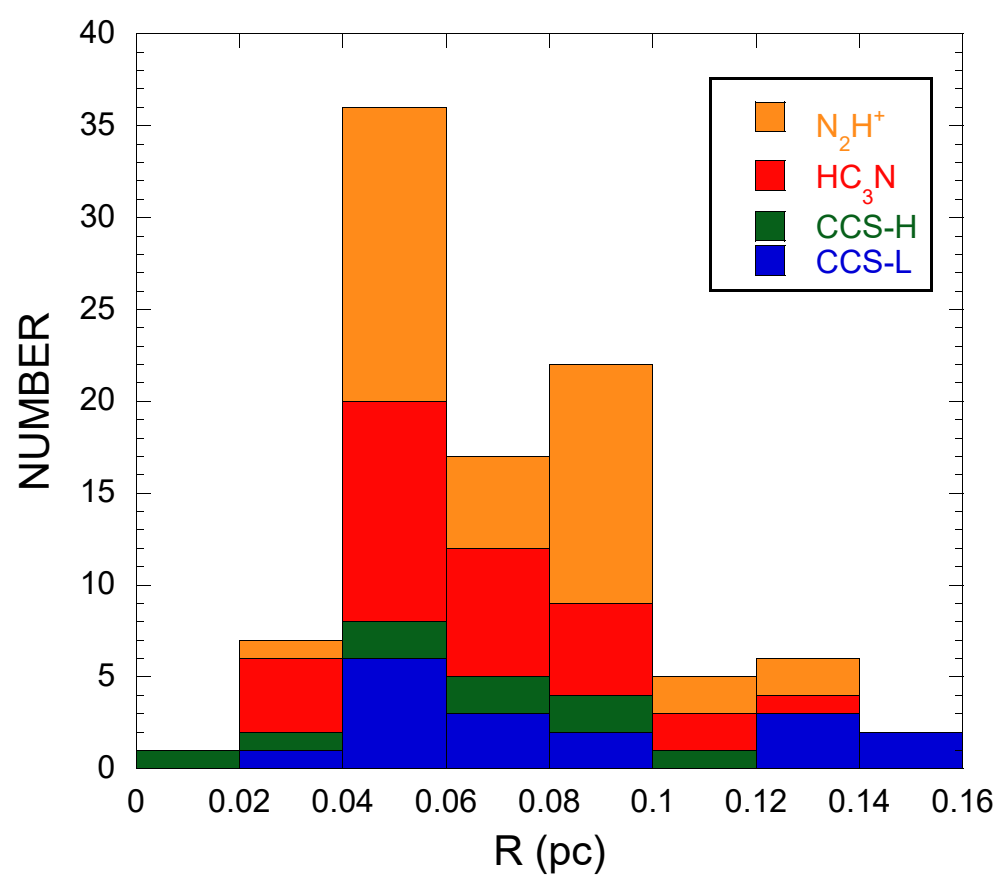

Figure 11. Histogram of the deconvolved radius $R$ for each line. Statistics are listed in Table 5 .

at the employed spatial resolution, as already noted by Kim et al. (2020). The absence of differences in the velocity dispersion between the starless and star-forming core contradicts the results of earlier studies (Beichman et al. 1986; Benson \& Myers 1989; Zhou et al. 1989; Tatematsu et al. 1993). One possibility is that largely improved sensitivities allow the identification of low-luminosity young stellar objects (YSOs), which were previously undetectable. To test this possibility, we plotted the nonthermal velocity dispersion against the bolometric luminosity of the YSO taken from Dutta et al. (2020) (Figure 12). Except for the core with the most luminous YSO (G211.47-19.27South, $L=180 \pm 70$ $\left.L_{\odot}\right)$, no clear positive correlation was observed. Our data were less affected by star formation activities because the tracer $\mathrm{N}_{2} \mathrm{H}^{+}$employed is insensitive to shocked gas (Turner \& Thaddeus 1977; Womack et al. 1993; Bachiller et al. 1996; Caselli et al. 2002), unlike CS and $\mathrm{NH}_{3}$ employed in previous studies. Our samples contain warm $T_{b o l}$ YSOs, which are not too young to observe the impacts of YSO activities.

We also compared the deconvolved radii (Yi et al. 2018) between the starless and star-forming cores. Figure 13 shows the histogram of the SCUBA-2 radius $R$ (dust) of the two categories. The starless and star-forming cores have very similar radii. We corrected the semi-major axis $a$ and semi-minor axis $b$ for the telescope beam in quadrature, and calculated the deconvolved radius $R$ :

$$
R=\left[\left(a^{2}-(\mathrm{HPBW} / 2)^{2}\right)\left(b^{2}-(\mathrm{HPBW} / 2)^{2}\right)\right]^{1 / 4} .
$$




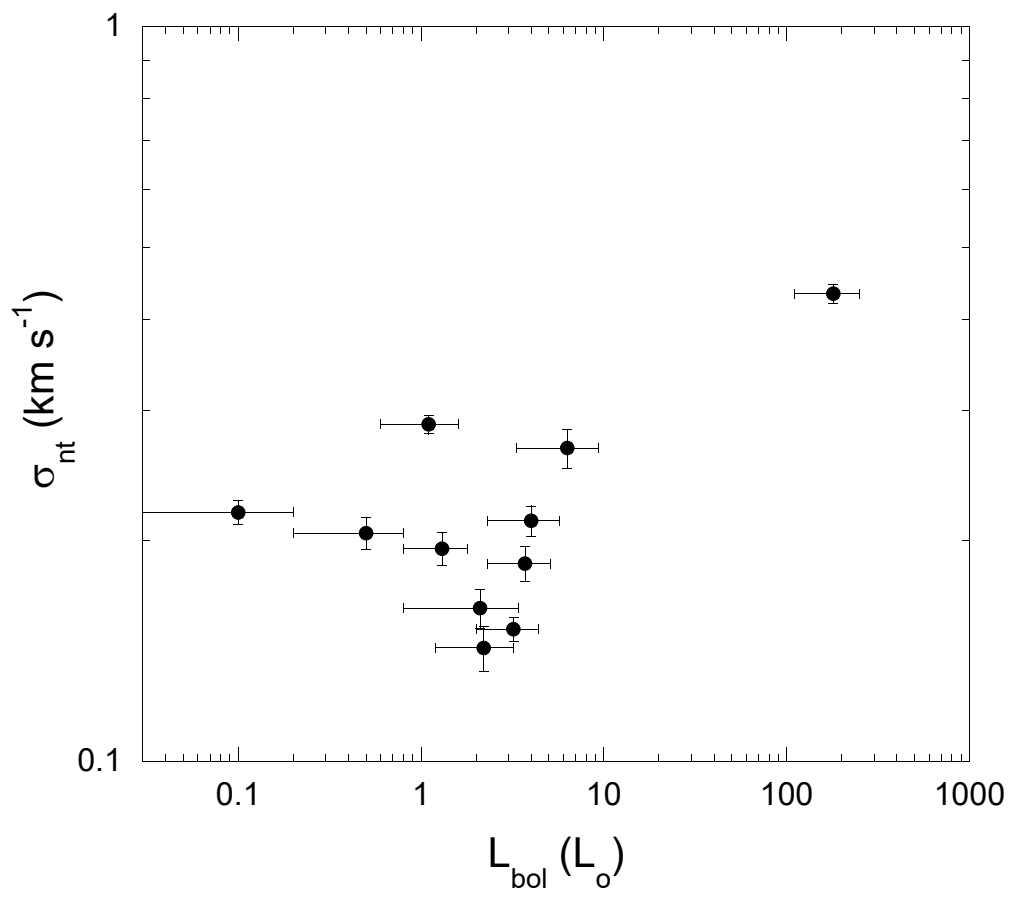

Figure 12. Nonthermal velocity dispersion vs. bolometric luminosity of the YSO (Dutta et al. 2020).

The SCUBA-2 beam radius is 7 arcsec or $0.0135 \mathrm{pc}$; thus, the cores are well resolved. This similarity is not consistent with the result from $\mathrm{H}^{13} \mathrm{CO}^{+}$observations toward Taurus by Mizuno et al. (1994), who concluded that starless cores are larger than star-forming cores, but consistent with that from $\mathrm{N}_{2} \mathrm{H}^{+}$observations by Tatematsu et al. (2004) for the same cores. A possible reason for this difference is the depletion of $\mathrm{H}^{13} \mathrm{CO}^{+}$molecules in the starless cores, which could cause flat-topped radial intensity profiles toward cold starless cores, and correspondingly lead to larger radii (Tatematsu et al. 2004). It seems that when we use a molecular-line tracer affected by depletion, we see a size difference between the starless and star-forming core. In contrast, when we use the dust continuum or a tracer that is less affected by depletion, it seems difficult to observe differences in radii with HPBWs of $0.01-0.03$ pc. When we adopt Welch's t-test for the null hypothesis that the starless and star-forming cores have equal radii, we obtain a p-value of 0.55 , which is considerably larger than a standard threshold of 0.05 for statistical significance. Thus, we cannot statistically conclude that these two categories have different radii.

It is suggested that sizes of identified cores are often very close to the telescope beam size, and this can be well explained if cores have power-law density profiles. Ladd et al. (1991) indicated that the observed emission size tends to be $10-80 \%$ larger than the original beam size if we assume the power-law radial intensity distribution. Young et al. (2003) showed anticorrelation between the power-law index and the deconvolved emission size (see their Fig. 27). Then, in such cases, the observed radius represents the radial density slope rather than the physical boundary. The mean HWHM radius $\left(23^{\prime \prime} 0 \pm 11^{\prime \prime} 4\right.$ or $\left.0.045 \pm 0.022 \mathrm{pc}\right)$ and beam radius $\left(7^{\prime \prime}\right.$ or $\left.0.0135 \mathrm{pc}\right)$ suggest a power-law index 


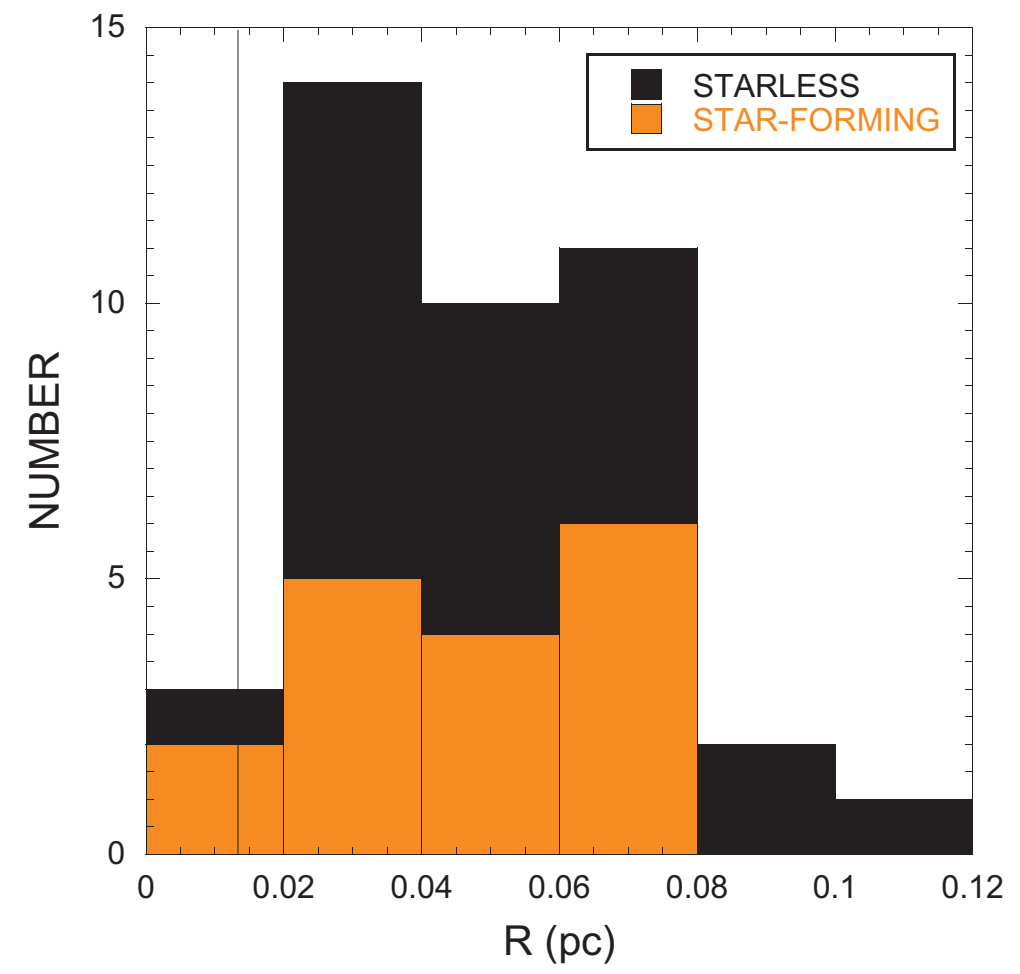

Figure 13. Histogram of the deconvolved radii of the SCUBA-2 cores in the Orion region. Black and orange colors indicate the starless and star-forming cores, respectively. The vertical solid line represents the telescope beam radius.

$p$ of $<1.4$ for the radial density profile $\rho(r) \propto r^{-p}$. Therefore, the SCUBA-2 core in Orion studied by Yi et al. (2018) arguably has a shallow radial density profile.

\section{DISCUSSION}

\subsection{Physical parameters of the starless cores against CEF2.0}

We investigated how the physical parameters of the starless cores compare to evolutionary indications based on chemistry, as encoded by CEF2.0, which is the chemical evolution tracer based the deuterium fraction. Table 6 lists the deuterium fraction and CEF2.0 for all cores which allow column density estimation. Note that CEF2.0 is defined only for the starless cores in Orion, but this Table contains both the starless and star-forming cores, and also the cores outside Orion. Kim et al. (2020) pointed out that the deuterium fraction will be seriously underestimated for distant $(>1 \mathrm{kpc})$ cores due to beam dilution. The adopted distance is listed in Table A1. Table A2 in the Appendix contains the YSO association information. The CEF2.0 values of the Orion starless cores were calculated from the column density ratios of $\mathrm{N}_{2} \mathrm{D}^{+} / \mathrm{N}_{2} \mathrm{H}^{+}$and $\mathrm{DNC} / \mathrm{HN}^{13} \mathrm{C}$ from our single-pointing observations (Kim et al. 2020). We question whether there is any evidence that stable cores change into unstable cores by changing physical properties such as turbulence.

Figure 14 shows the plot of the nonthermal velocity dispersions, radii, $\mathrm{H}_{2}$ column densities, masses, and virial parameters of the Orion starless cores against CEF2.0, in which no significant trend is observed. Radius $R$ and mass 


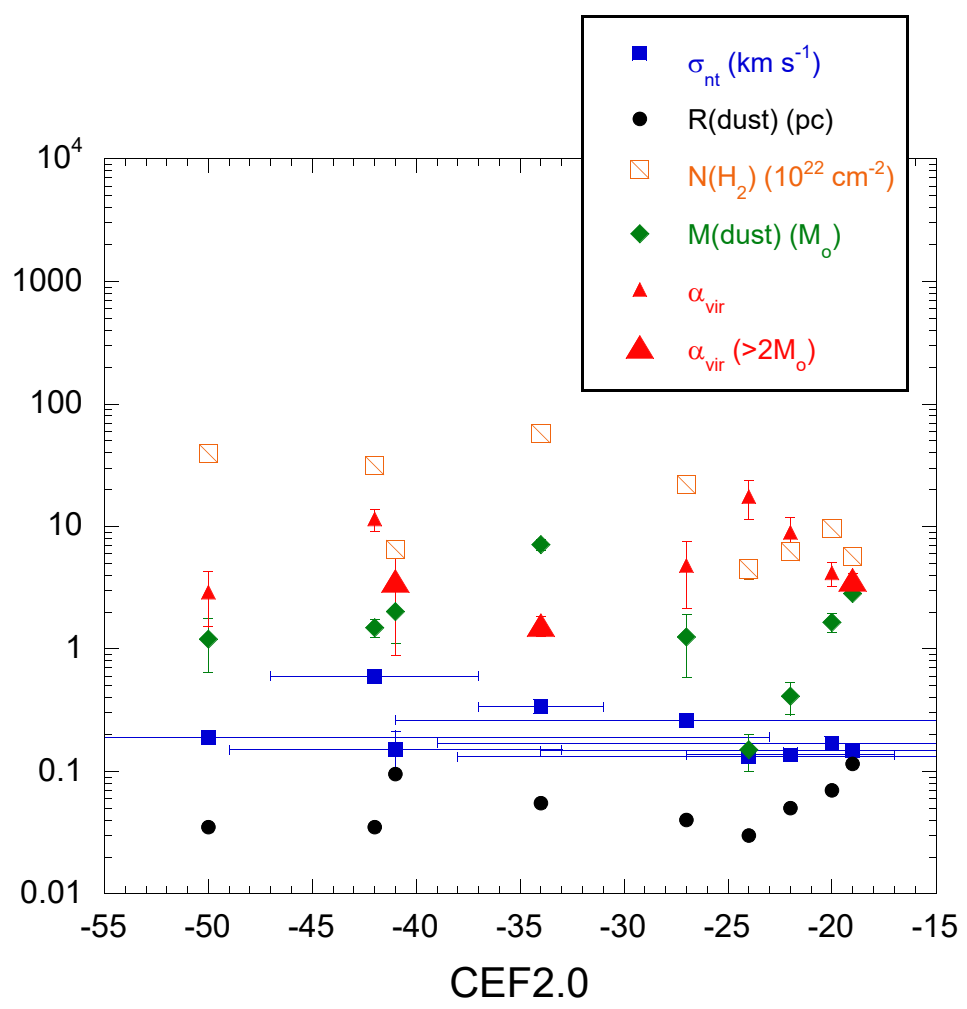

Figure 14. Nonthermal velocity dispersions, radii, $\mathrm{H}_{2}$ column densities, masses, and virial parameters of the starless core vs. CEF2.0. The core radii, column densities, and masses are taken from Yi et al. (2018). The error bar for CEF2.0 is plotted only for the nonthermal velocity dispersion for clarity.

$M$ increase with increasing CEF2.0 from CEF2.0 $=-24$ to -19 , but this rise is probably not significant when we take into account the error in CEF2.0. The virial parameter $\alpha_{v i r}$ of the starless cores is as large as $1-20$. If we allow $1 \sigma$ uncertainty, four cores (44\%) out of the nine starless cores in this figure may fit the range $0.5<\alpha_{v i r}<2$. Large virial parameters for starless cores suggest that most of them are either pressure-confined or transient. Chen et al. (2020) showed the evolution of unbound cores into bound ones in their simulation.

Crapsi et al. (2005) noted that the chemical properties (including the deuterium fraction) and physical properties (density, line width, etc.) of starless cores as a whole can trace the evolutionary stage, although they did not find very tight dependencies between these properties. Their samples included starless cores at younger stages such as B68, TMC-1, L492, L1498, and L1512 (Shirley et al. 2005; Hirota \& Yamamoto 2006), which have values of -100 $\lesssim$ CEF2.0 $\lesssim-60$ (Kim et al. 2020), implying lower deuterium fractions. They concluded that evolved starless cores with higher $N\left(\mathrm{~N}_{2} \mathrm{D}^{+}\right) / N\left(\mathrm{~N}_{2} \mathrm{H}^{+}\right)$tend to have higher central $\mathrm{H}_{2}$ column densities and more compact density profiles. They employed multiple tracers including $\mathrm{N}_{2} \mathrm{H}^{+}, \mathrm{N}_{2} \mathrm{D}^{+}$, and the dust continuum emission, and the above physical parameters were derived at linear resolutions of $0.006-0.02 \mathrm{pc}, 1.5-6$ times better than our linear resolution $(0.03-0.04$ pc). The difference in the linear resolution and/or the CEF2.0 range may at least partially explain the difference in conclusion. 
Marka et al. (2012) observed Bok globules in $\mathrm{NH}_{3}$ and CCS to study their chemical evolution. They did not find any globules with extremely high CCS abundances and concluded that all of the observed Bok globules are in a relatively evolved chemical state. It is possible that SCUBA-2 cores with high column densities selected in our study are similarly biased to evolved cores.

We did not observe intense CCS emission even at the starless core stage. Relatively late chemical evolution stages may be one reason, but we also suspect that serious depletion occurs in CCS at cold starless cores, which leads to low values of the CCS column density. Some previous studies of core chemistry evolution used the abundance at the molecular line emission maximum (Tatematsu et al. 2014; Ohashi et al. 2014), which can be different from that at density peaks traced by the dust continuum emission. In such cases, depletion of CCS can be alleviated. We used the SCUBA-2 position as the core centers where CCS depletion could be severe.

\subsection{Core evolution against the bolometric temperature}

We investigated the physical parameters of the star-forming cores against the bolometric temperature of associated YSOs (Dutta et al. 2020). The bolometric temperature is a reliable empirical tracer of a star-forming core's evolution (Chen et al. 1995). The bolometric temperature $T_{b o l}$ can be obtained from the flux-weighted mean frequencies in the observed spectral energy distributions (SEDs) (Myers \& Ladd 1993). Dutta et al. (2020) used their ALMA $1.3 \mathrm{~mm}$ fluxes as well as fluxes at other wavelengths from Cutri et al. (2003), Lawrence et al. (2007), Wright et al. (2010), Megeath et al. (2012), Stutz et al. (2013), Tobin et al. (2015), Doi et al. (2015), and Yi et al. (2018). Figure 15 shows the nonthermal velocity dispersions, radii, $\mathrm{H}_{2}$ column densities, masses, and virial parameters vs. $T_{b o l}$ for the star-forming cores (Dutta et al. 2020). We did not observe any significant trends in this Figure. The virial parameter $\alpha_{v i r}$ of the star-forming core ranged from 0.1 to 20. Six (55\%) of the eleven star-forming cores in this Figure may fit the range $\alpha_{v i r}=0.5-2$ when we allow a $1 \sigma$ uncertainty. The core that forms a protostar should be gravitationally bound or pressure confined at least when the protostar forms. Large virial parameters of the star-forming core suggest either the confining external pressure, fast core dispersal after star formation, strong tidal forces from the ambient gas, or systematic bias in estimation of the parameter. Furthermore, the associated outflow can stir up the core to some extent through magnetic fields, although $\mathrm{N}_{2} \mathrm{H}^{+}$is less affected by shocks.

We also investigated the distribution of CCS (young-gas tracer) and $\mathrm{N}_{2} \mathrm{H}^{+}$(evolved-gas tracer) vs. $T_{b o l}$. Figure 16 shows the close-up maps of representative star-forming cores aligned in order of the bolometric temperature $T_{b o l}$ of the associated YSO as an evolutionary tracer. Comparisons including $\mathrm{HC}_{3} \mathrm{~N}$ on the full-sized maps of the all star-forming Orion cores with $T_{b o l}$ can be seen in Table A2, Figures1 to 7, and Figure Set 17. Again, no clear trend is seen.

\subsection{Relationship with the association with the Filament}

Some of the observed cores are apparently located in filaments. Because filaments likely play an important role in core mass accretion, we investigate whether there are any differences between cores inside or outside filaments. Association with a filament was judged from the SCUBA-2 and $\mathrm{N}_{2} \mathrm{H}^{+}$distributions, and is shown in Table 4. Table 7 summarizes the statistics of the Orion core. Here, we do not distinguish the starless and star-forming cores. The nonthermal velocity dispersions and radii are similar between the two categories. The cores associated with filaments are slightly less massive and have smaller virial parameters, although the standard deviation is large and so the differences may not be significant.

\subsection{Kinematics}

Details of the kinematics of the cores discussed here are beyond the scope of this paper, but we consider here two example fields, each containing representative examples, G206.12 and G211.16. We selected them from Orion cores, as they have more accurate distances (Kounkel et al. 2017; Perryman et al. 1997) and are less affected by beam dilution (Kim et al. 2020).

One field G206.12 has only one SCUBA-2 core inside the mapped area (Figure 2), whereas the other field G211.16 contains six SCUBA-2 cores (Figure 5). The $\mathrm{N}_{2} \mathrm{H}^{+}$emission was used for analysis. We calculated the intensityweighted radial velocity (moment 1) using the hyperfine group $J=1 \rightarrow 0 F_{1}=2 \rightarrow 1$ containing the brightest hyperfine component to obtain better $\mathrm{S} / \mathrm{N}$ ratios, and the velocity dispersion (moment 2 ) using the isolated hyperfine component $J=1 \rightarrow 0, F_{1}, F=0,1 \rightarrow 1,2$, which is optically thinner and less affected by the neighboring satellites.

Field G206.12 (Figure 17) does not exhibit any significant velocity gradients. The velocity dispersion seems to increase toward the core center harboring the YSO. This shift may represent the influence from the YSO. In field G211.16 (Figure 18), however, the velocity field shows distinct velocities for filaments. 
Table 6. Deuterium Fraction and CEF2.0

\begin{tabular}{|c|c|c|c|c|c|}
\hline SCUBA-2 core & $\begin{array}{c}V_{L S R} \\
\mathrm{~km} \mathrm{~s}^{-1}\end{array}$ & $N\left(\mathrm{~N}_{2} \mathrm{D}^{+}\right) / N\left(\mathrm{~N}_{2} \mathrm{H}^{+}\right)$ & $N(\mathrm{DNC}) / N\left(\mathrm{HN}^{13} \mathrm{C}\right)$ & YSO & CEF 2.0 \\
\hline G192.32-11.88North & 12.1 & $0.15 \pm 0.08$ & $5.2 \pm 3.7$ & $\mathrm{Y}$ & \\
\hline G192.32-11.88South & 12.2 & $0.17 \pm 0.02$ & $3.8 \pm 2.7$ & Y & \\
\hline G203.21-11.20West1 & 10.7 & $0.30 \pm 0.04$ & $<4.1$ & $\mathrm{Y}$ & \\
\hline G203.21-11.20West2 & 10.2 & $0.21 \pm 0.05$ & $<4.0$ & Y & \\
\hline G204.4-11.3A2East & 1.6 & $0.27 \pm 0.07$ & $<4.2$ & $\mathrm{Y}$ & \\
\hline G204.4-11.3A2West & 1.7 & $0.09 \pm 0.04$ & $\ldots$ & Y & \\
\hline G206.12-15.76 & 8.5 & $\ldots$ & $<4.8$ & Y & \\
\hline G206.93-16.61West1 & 9.3 & $<0.15$ & $1.0 \pm 0.7$ & Y & \\
\hline G206.93-16.61West3 & 9.3 & $0.04 \pm 0.02$ & $1.7 \pm 1.2$ & $\mathrm{Y}$ & \\
\hline G206.93-16.61West4 & 10.1 & $0.16 \pm 0.04$ & $5.1 \pm 3.7$ & $\mathrm{~N}$ & $-27 \pm 14$ \\
\hline G206.93-16.61West5 & 9.0 & $0.15 \pm 0.02$ & $\ldots$ & $\mathrm{N}$ & $-34 \pm 3$ \\
\hline G206.93-16.61West6 & 10.4 & $<0.23$ & $2.7 \pm 1.9$ & $\mathrm{Y}$ & \\
\hline G207.36-19.82North2 & 11.2 & $0.22 \pm 0.05$ & $<6.8$ & $\mathrm{Y}$ & \\
\hline G207.36-19.82South & 11.3 & $0.11 \pm 0.04$ & $\ldots$ & $\mathrm{N}$ & $-41 \pm 8$ \\
\hline G208.68-19.20North1 & 11.1 & $<0.08$ & $2.6 \pm 1.8$ & $\mathrm{Y}$ & \\
\hline G208.68-19.20North2 & 11.2 & $0.11 \pm 0.01$ & $3.1 \pm 2.2$ & $\mathrm{Y}$ & \\
\hline G208.68-19.20North3 & 11.1 & $0.06 \pm 0.01$ & $2.7 \pm 1.9$ & $\mathrm{Y}$ & \\
\hline G209.05-19.73North & 8.3 & $0.27 \pm 0.06$ & $5.7 \pm 4.2$ & $\mathrm{~N}$ & $-19 \pm 15$ \\
\hline G209.05-19.73South & 7.9 & $0.23 \pm 0.13$ & $5.9 \pm 4.4$ & $\mathrm{~N}$ & $-20 \pm 19$ \\
\hline G209.29-19.65North1 & 8.6 & $0.06 \pm 0.03$ & $2.0 \pm 1.4$ & $\mathrm{~N}$ & $-58 \pm 17$ \\
\hline G209.29-19.65South1 & 7.5 & $0.11 \pm 0.02$ & $\ldots$ & $\mathrm{N}$ & $-42 \pm 5$ \\
\hline G209.29-19.65South2 & 7.8 & $<0.08$ & $3.4 \pm 2.5$ & $\mathrm{~N}$ & $-38 \pm 36$ \\
\hline G209.29-19.65South2 & 9.0 & $0.14 \pm 0.08$ & $0.7 \pm 0.5$ & $\mathrm{~N}$ & $-67 \pm 18$ \\
\hline G209.77-19.40East1 & 8.1 & $0.05 \pm 0.01$ & $1.8 \pm 1.3$ & $\mathrm{Y}$ & \\
\hline G209.77-19.40East2 & 8.0 & $<0.14$ & $2.5 \pm 1.8$ & $\mathrm{~N}$ & $-50 \pm 27$ \\
\hline G209.77-19.40East3 & 7.8 & $0.25 \pm 0.04$ & $4.7 \pm 3.4$ & $\mathrm{~N}$ & $-23 \pm 14$ \\
\hline G209.77-19.40East3 & 8.3 & $<0.63$ & $1.9 \pm 1.4$ & $\mathrm{~N}$ & $-61 \pm 28$ \\
\hline G209.94-19.52North & 8.1 & $0.33 \pm 0.07$ & $3.6 \pm 2.5$ & $\mathrm{Y}$ & \\
\hline G209.94-19.52South1 & 7.5 & $0.24 \pm 0.13$ & $6.3 \pm 4.5$ & $\mathrm{~N}$ & $-18 \pm 15$ \\
\hline G209.94-19.52South1 & 8.1 & $0.24 \pm 0.03$ & $4.1 \pm 2.9$ & $\mathrm{~N}$ & $-26 \pm 14$ \\
\hline G210.82-19.47North1 & 5.3 & $0.32 \pm 0.03$ & $4.0 \pm 2.9$ & $\mathrm{Y}$ & \\
\hline G210.82-19.47North2 & 5.3 & $0.20 \pm 0.02$ & $5.3 \pm 3.8$ & $\mathrm{~N}$ & $-24 \pm 14$ \\
\hline G211.16-19.33North2 & 3.7 & $0.10 \pm 0.04$ & $<2.5$ & $\mathrm{Y}$ & \\
\hline G211.16-19.33North3 & 3.4 & $0.24 \pm 0.05$ & $<3.5$ & $\mathrm{~N}$ & $-22 \pm 5$ \\
\hline G211.16-19.33North5 & 4.3 & $0.31 \pm 0.06$ & $<3.2$ & $\mathrm{Y}$ & \\
\hline G211.47-19.27North & 4.1 & $0.10 \pm 0.02$ & $<3.1$ & $\mathrm{Y}$ & \\
\hline G212.10-19.15North1 & 4.3 & $0.35 \pm 0.07$ & $<6.1$ & $\mathrm{Y}$ & \\
\hline G212.10-19.15North3 & 4.2 & $0.06 \pm 0.03$ & $2.8 \pm 2.0$ & $\mathrm{Y}$ & \\
\hline G212.10-19.15South & 3.9 & $0.39 \pm 0.06$ & $<3.5$ & $\mathrm{Y}$ & \\
\hline SCOPEG159.18-20.09 & 6.3 & $\ldots$ & $13.8 \pm 9.7$ & $\mathrm{Y}$ & \\
\hline SCOPEG159.22-20.11 & 6.7 & $\ldots$ & $10.5 \pm 7.5$ & $\mathrm{Y}$ & \\
\hline SCOPEG173.17+02.36 & -18.9 & $\ldots$ & $1.1 \pm 0.8$ & Y & \\
\hline SCOPEG173.18+02.35 & -19.0 & . . & $1.3 \pm 0.9$ & $\mathrm{Y}$ & \\
\hline SCOPEG173.19+02.35 & -19.3 & . . & $1.6 \pm 1.2$ & $\mathrm{Y}$ & \\
\hline SCOPEG001.37+20.95 & 0.8 & $\ldots$ & $14.6 \pm 10.3$ & $\mathrm{~N}$ & \\
\hline SCOPEG017.38+02.26 & 10.7 & $\ldots$ & $1.7 \pm 1.3$ & $\mathrm{Y}$ & \\
\hline SCOPEG017.37+02.24 & 10.5 & $\ldots$ & $1.1 \pm 0.8$ & $\mathrm{Y}$ & \\
\hline SCOPEG017.36+02.23 & 10.4 & $\ldots$ & $0.6 \pm 0.4$ & $\mathrm{Y}$ & \\
\hline SCOPEG014.18-00.23 & 40.5 & $\ldots$ & $1.1 \pm 0.8$ & Y & \\
\hline SCOPEG016.93+00.25 & 24.2 & . . & $2.3 \pm 1.6$ & $\mathrm{~N}$ & \\
\hline SCOPEG016.93+00.24 & 24.3 & $\ldots$ & $0.6 \pm 0.4$ & $\mathrm{~N}$ & \\
\hline SCOPEG016.93+00.24 & 26.3 & $\ldots$ & $0.8 \pm 0.6$ & $\mathrm{~N}$ & \\
\hline SCOPEG016.93+00.22 & 23.6 & $\ldots$ & $1.4 \pm 1.0$ & $\mathrm{Y}$ & \\
\hline SCOPEG033.74-00.01 & 105.0 & . . & $0.4 \pm 0.3$ & $\mathrm{Y}$ & \\
\hline SCOPEG035.48-00.29 & 45.3 & $\ldots$ & $1.8 \pm 1.3$ & Y & \\
\hline SCOPEG035.52-00.27 & 45.1 & . . . & $1.6 \pm 1.1$ & $\mathrm{Y}$ & \\
\hline SCOPEG035.48-00.31 & 44.9 & $\ldots$ & $1.6 \pm 1.1$ & $\mathrm{Y}$ & \\
\hline SCOPEG034.75-01.38 & 45.6 & $\ldots$ & $0.9 \pm 0.6$ & $\mathrm{Y}$ & \\
\hline SCOPEG069.80-01.67 & 12.4 & $\ldots$ & $1.2 \pm 0.8$ & $\mathrm{Y}$ & \\
\hline SCOPEG069.81-01.67 & 12.1 & $\ldots$ & $1.2 \pm 0.9$ & $\mathrm{Y}$ & \\
\hline SCOPEG105.37+09.84 & -9.7 & . . & $1.8 \pm 1.3$ & Y & \\
\hline SCOPEG107.16+05.45 & -10.2 & $\ldots$ & $5.5 \pm 3.9$ & Y & \\
\hline SCOPEG107.18+05.43 & -10.8 & $\ldots$ & $1.8 \pm 1.3$ & $\mathrm{Y}$ & \\
\hline
\end{tabular}




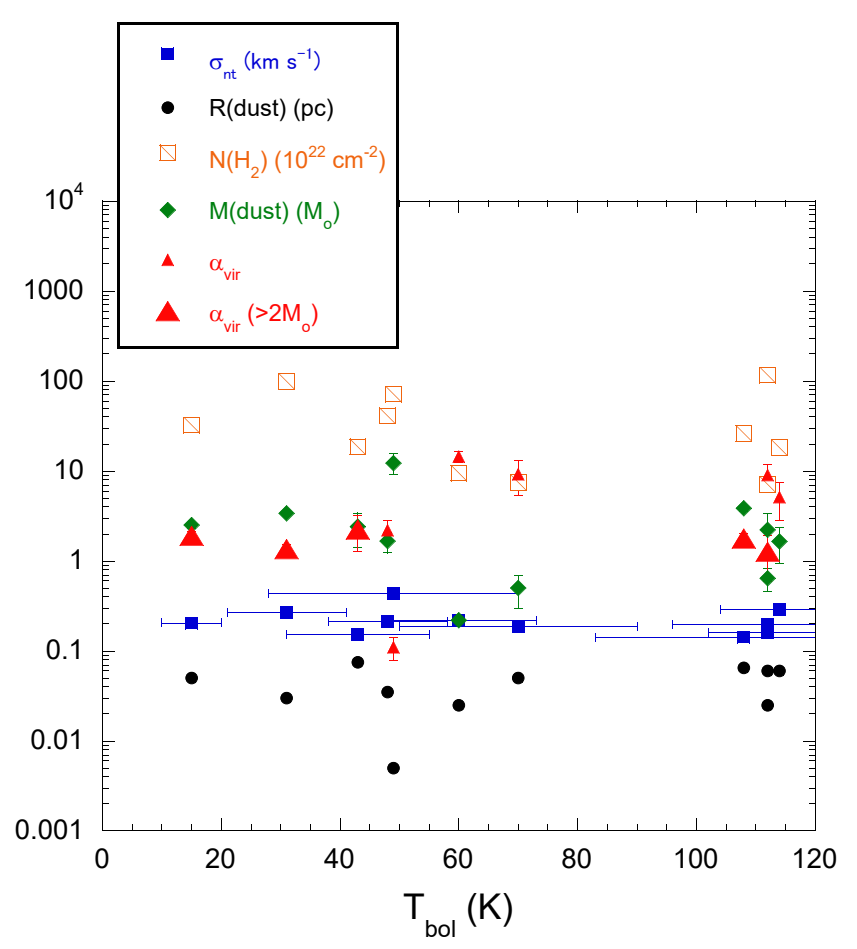

Figure 15. Nonthermal velocity dispersions, radii, $\mathrm{H}_{2}$ column densities, masses, and virial parameters of the star-forming cores vs. bolometric temperature $T_{b o l}$ of the YSOs (Dutta et al. 2020). The error bar for $T_{b o l}$ is plotted only for the nonthermal velocity dispersion for clarity.

Table 7. Core Properties with and without the filaments

\begin{tabular}{|c|c|c|c|c|c|}
\hline Filament & Calculation & $\begin{array}{c}\sigma_{n t} \\
\mathrm{~km} \mathrm{~s}^{-1}\end{array}$ & $\begin{array}{c}R \text { (dust) } \\
\text { pc }\end{array}$ & $\begin{array}{c}M \text { (dust) } \\
M_{\odot}\end{array}$ & $\alpha_{v i r}$ \\
\hline Y & mean & $0.24 \pm 0.11$ & $0.05 \pm 0.02$ & $1.58 \pm 1.41$ & $6.29 \pm 4.56$ \\
\hline Y & median & 0.20 & 0.05 & 1.23 & 4.93 \\
\hline $\mathrm{N}$ & mean & $0.25 \pm 0.09$ & $0.04 \pm 0.03$ & $3.03 \pm 3.66$ & $4.54 \pm 4.62$ \\
\hline $\mathrm{N}$ & median & 0.22 & 0.04 & 2.01 & 3.18 \\
\hline
\end{tabular}

\section{SUMMARY}

We performed OTF mapping observations of 44 fields containing 107 SCUBA-2 cores in the $\mathrm{N}_{2} \mathrm{H}^{+}, \mathrm{HC}_{3} \mathrm{~N}, 94 \mathrm{GHz}$ CCS, and $82 \mathrm{GHz}$ CCS lines using the Nobeyama 45-m telescope. The distribution of $\mathrm{N}_{2} \mathrm{H}^{+}$and $\mathrm{HC}_{3} \mathrm{~N}$ line emissions is similar to the respective $850-\mu \mathrm{m}$ distribution, whereas CCS lines are undetected or distributed in clumpy structures surrounding the peak position of the $850-\mu \mathrm{m}$ dust continuum emission. Occasionally (12\%), we detected the CCS emission, which is considered an early-type gas tracer, toward YSOs. The nonthermal velocity dispersions of the SCUBA-2 cores in Orion are very similar between the starless and star-forming core populations, suggesting that evolution toward star formation does not immediately affect nonthermal velocity dispersion. 

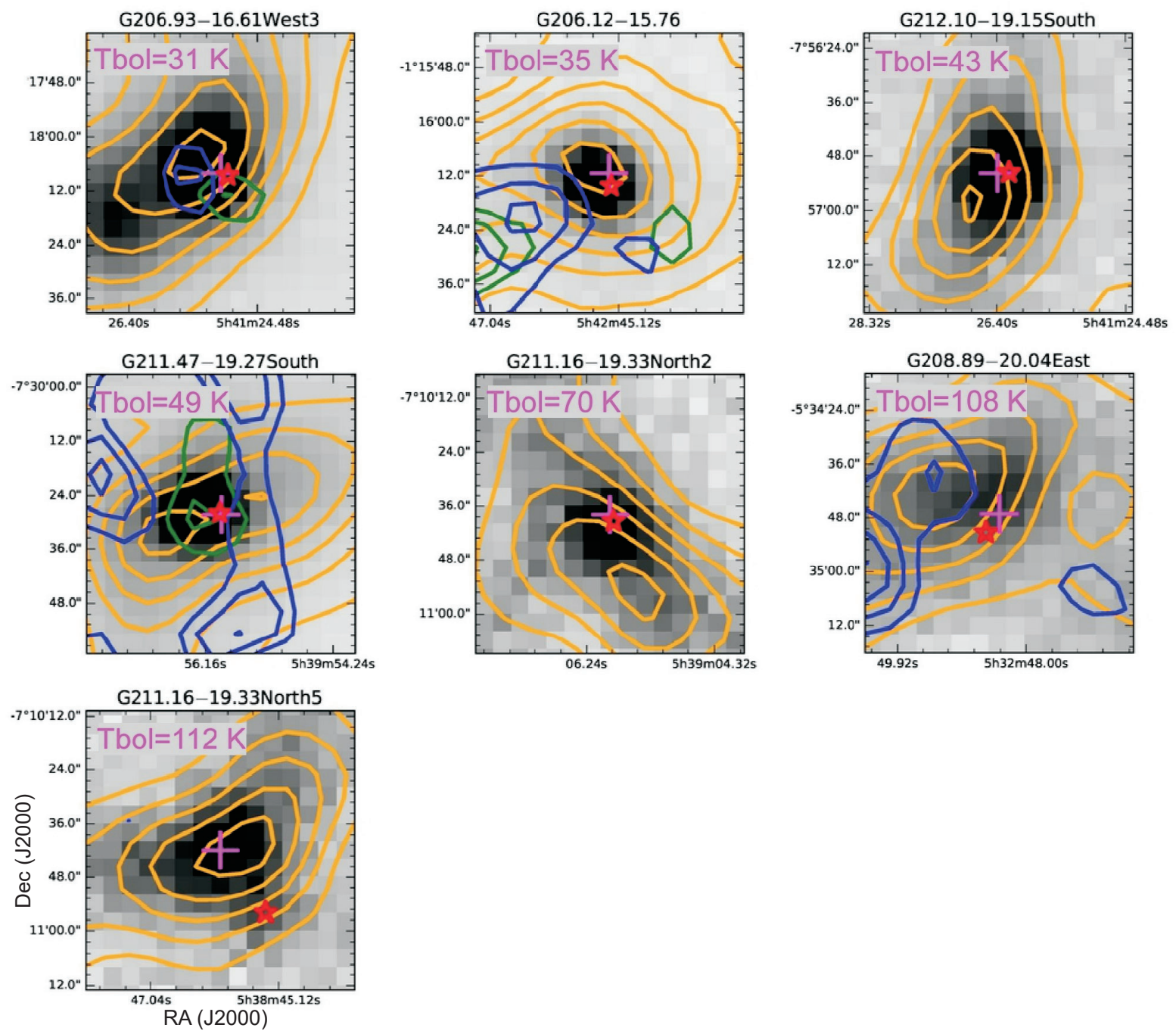

Figure 16. Close-up maps sorted in order of evolutionary stages using $T_{\text {bol }}$ for representative star-forming cores. Each map represents the integrated intensity contour map superposed on the $850-\mu$ m dust continuum emission map. The orange, green, and blue contours represent the $\mathrm{N}_{2} \mathrm{H}^{+}, \mathrm{CCS}-\mathrm{H}$, and CCS-L integrated-intensity contour maps, respectively. The innermost to outermost contour levels correspond to $95 \%, 80 \%, 65 \%, 50 \%, 35 \%, 20 \%, 5 \%$, and $3 \sigma$, respectively. The peak value and $\sigma$ value of integrated intensity are listed in Table A3. The cross and star symbols represent a SCUBA-2 core and a protostar, respectively. The error for $T_{\mathrm{bol}}$ is listed in Table A2 in the Appendix.

\section{REFERENCES}

Aikawa Y., Ohashi, N., Inutsuka, S., Herbst, E., \&

Takakuwa, S. 2001, ApJ, 552, 639

André, P., Ward-Thompson, D., \& Motte, F. 1996, A\&A, 314,625

Astropy Collaboration, Robitaille, T. P., Tollerud, E. J., et al. 2013, A\&A, 558, A33

Astropy Collaboration, Price-Whelan, A. M., Sipőcz, B. M., et al. 2018, AJ, 156, 123

Bachiller, R. 1996, ARA\&A, 34, 111

Beichman, C. A., Myers, P. C., Emerson, J. P., et al. 1986, ApJ307, 337
Benson, P. J., \& Myers, P. C. 1989, ApJS, 71, 89

Benson, P. J., Caselli, P., \& Myers, P. C. 1998, ApJ, 506, 743

Bertoldi, F. \& McKee, C. F. 1992, ApJ, 395, 140

Brünken, S., Sipilä, O., Chambers, E. T., et al. 2014, Nature, 516, 219

Caselli, P., Walmsley, C. M., Tafalla, M., et al. 1999, ApJ, 523, L165

Caselli, P., Myers, P. C., \& Thaddeus, P. 1995, ApJ, 455, L77 

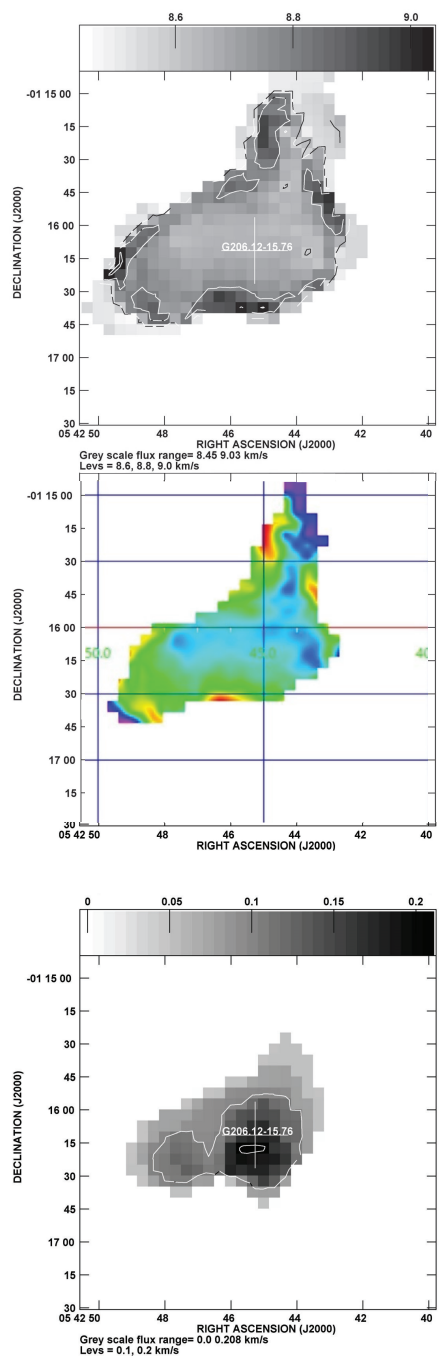

Figure 17. The top and middle panels show the intensity-weighted velocity (moment 1) maps in grey-scale and in pseudo-color, respectively, toward field G206.12 (Figure 2). The bottom panel shows the intensity-weighted velocity dispersion (moment 2) map in grey-scale toward the same field. 

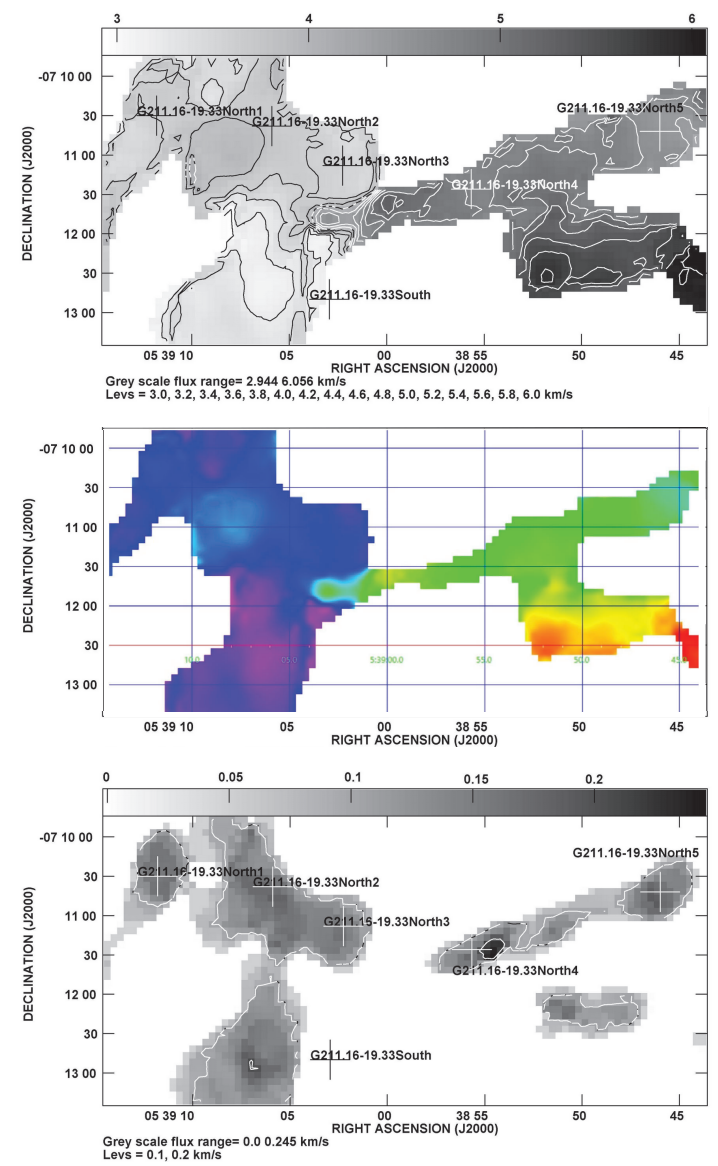

Figure 18. The same as Figure 17 but for field G211.16 (Figure 5). 
Caselli, P.,Benson, P. J., Myers, P. C., et al. 2002, ApJ, 572,238

Chen, H., Myers, P. C., Ladd, E. F., \& Wood, D. O. S. 1995, ApJ, 445, 377

Chen, H. H.-H., Offner, S. S. R., Pineda, J. E., et al. https://arxiv.org/abs/2006.07325

Crapsi, A., Caselli, P., Walmsley, C. M., et al. 2005, ApJ, 619,379

Cummins, S. E., Linke, R. A., \& Thaddeus, P. 1986, ApJS, 60,819

Cutri, R. M., Skrutskie, M. F., van Dyk, S., et al. 2003, yCat, II $/ 246$

Doi, Y., Takita, S., Ootsubo, T., et al. 2015, PASJ, 67, 50

Dutta, S., Lee, C.-F., Hirano, N., et al. 2020, ApJS, 251, 20

Eden, D. J., Liu, T., Kim, K.-T., et al. 2019, MNRAS, 485, 2895

Emprechtinger, M., Caselli, P., Volgenau, N. H., Stutzki, J., \& Wiedner, M. C. 2009, A\&A, 493, 89

Fehér, O., Tóth, L. V., Kraus A., et al. 2020, ApJS, submitted

Feng, S., Caselli, P., Wang, K., et al. 2019, ApJ, 883, 202

Feng, S., Li, D., Caselli, P., et al. 2020, ApJ, 901, 145

Fontani, F., Palau, A., Caselli, P., et al. 2011, A\&A, 529, L7

Fuller, G. A., \& Myers, P. C. 1992, ApJ, 384, 523

Gerner, T., Shirley, Y. L., Beuther, H., et al. 2015, A\&A, 579, A 80

Ge, J. X., Mardones, D., He, J. H., et al., ApJ, 891, 36

Getman, K. V., Feigelson, E. D., Kuhn, M. A., \& Garmire, G. P. 2019, MNRAS, 487, 2977

Gómez, G. C., Vazquez Semadeni, E., Shadmehri, M., \& Ballesteros Paredes, J. 2007, ApJ, 669, 1042

Hily-Blant, P., Pineau des Forêts, G., Faure, A. \& Flower, D. R. 2020, A\&A, 643. 76

Hirahara, Y., Suzuki, H., Yamamoto, S., et al. 1992, ApJ, 394,539

Hirota, T., \& Yamamoto, S. 2006, ApJ, 646, 258

Jørgensen, J. K., Hogerheijde, M. R., van Dishoeck, E. F., Blake, G. A., \& Schöier, F. L., A\&A, 413, 993

Joye, W. A., \& Mandel, E. 2003, in A. S. P. Conf. Ser. 101, Astronomical Data Analysis Software and Systems V, ed. H. E. Payne, R. I. Jedrzejewski, \& R. N. Hook (San Franciso, CA: ASP), 489

Kamazaki, T., Okumura, S. K., Chikada, Y., et al. 2012, PASJ, 64, 29

Kerr, R., Kirk, H., Di Francesco, J., et al., ApJ, 874, 147

Kim, G., Tatematsu, K., Liu, T., et al. 2020, ApJS, 249, 33

Kirk, H., Friesen, R. K., Pineda, J. E., et al., ApJ, 846, 144

Kounkel, M., Hartmann, L., Loinard, L., et al. 2017, ApJ, 834,142
Ladd, E. F., Adams, F. C., Casey, S., et al. 1991, ApJ, 382, 555

Lawrence, A., Warren, S. J., Almaini, O., et al. 2007, MNRAS, 379, 1599

Lee, J.-E., Bergin, E. A., \& Evans, N. J. I. 2004, ApJ, 617, 360

Lee, J.-E., Evans, N. J. I., Shirley, Y. L., \& Tatematsu, K. 2003, ApJ, 583, 789

Li, Z.-Y., Shematovich, V. I., Wiebe, D. S., \& Shustov, B. M. 2002, ApJ, 569, 792

Liu, T., Wu, Y., Mardones, D., et al. 2015, Publications of The Korean Astronomical Society, 30, 79

Liu, T., Kim, K.-T., Juvela, M., et al. 2018, ApJS, 234, 28

Mao, S. A., Ostriker, E. C., \& Kim, C.-G. 2020 A\&A, 898, 52

Marka, C., Schreyer, K., Launhardt, R., Semenov, D. A. \& Henning, Th. 2012 A\&A, 537, A4

McKee, C. F., \& Zweibel, E. G. 1992, ApJ, 399, 551

Megeath, S. T., Gutermuth, R., Muzerolle, J., et al. 2012, AJ, 144, 192

Minamidani, T., Nishimura, A., Miyamoto, Y., et al. 2016, Millimeter, Submillimeter, and Far-Infrared Detectors and Instrumentation for Astronomy VIII, 9914, 99141Z

Mizuno, A., Onishi, T., Hayashi, M., et al. 1994, Nature, 368,719

Myers, P. C., \& Ladd, E. F. 1993, ApJ, 413, L47

Nakano, T. 1998, ApJ, 494, 587

Ohashi, S., Tatematsu, K., Choi, M., et al. 2014, PASJ, 66, 119

Ohashi, S., Tatematsu, K., Fujii, K., et al. 2016, PASJ, 68, 3

Onishi, T., Mizuno, A., Kawamura, A., Tachihara, K., \& Fukui, Y. 2002, ApJ, 575, 950

Pagani, L., Lesaffre, P., Jorfi, M., et al. 2013, A\&A, 551, 38

Perryman, M. A. C., Lindegren, L., Kovalevsky, J., et al. 1997, A\&A, 323, L49

Pickett, H. M., Poynter, R. L., Cohen, E. A., \& and, M. D. S. 1998, JQSRT, 60, 883

Planck Collaboration, Ade, P. A. R., Aghanim, N., et al. 2011, A\&A, 536, A23

Planck Collaboration, Ade, P. A. R., Aghanim, N., et al. 2016, A\&A, 594, A28

Redaelli, E., Bizzocchi, L., \& Caselli, P. 2020, A\&A, 644, 29

Reid, M. J., Dame, T. M., Menten, K. M., \& Brunthaler, A. 2016, ApJ, 823, 77

Robitaille, T., \& Bressert, E. 2012, Astrophysics Source Code Library, record ascl:1208.017

Sahu, D., Liu, S.-Y., Liu, T., et al. 2021, ApJ, 907, L15

Sakai, T., Sakai, N., Furuya, K., et al. 2012, ApJ, 747, 140

Sakai, T., Sakai, N., Furuya, K., et al. 2015, ApJ, 803, 70 
Savage, C., Apponi, A. J., Ziurys, L. M., et al. 2002, ApJ, 578,211

Sawada, T., Ikeda, N., Sunada, K., et al. 2008, PASJ, 60, 445

Shirley, Y. L. 2015, PASP, 127, 299

Shirley, Y. L., Nordhaus, M. K., Grcevich, J. M., et al. 2005, ApJ, 632, 982

Stutz, A. M., Tobin, J. J., Stanke, T., et al. 2013, ApJ, 767, 36

Suzuki, H., Yamamoto, S., Ohishi, M., et al. 1992, ApJ, 392,551

Tafalla, M., Myers, P. C., Caselli, P., Walmsley, C. M. \& Comito, C. 2002, ApJ, 569, 815

Tatematsu, K., Umemoto, T., Kameya, O., et al. 1993, ApJ, 404, 643

Tatematsu, K., Umemoto, T., Kandori, R., \& Sekimoto, Y. 2004, 606, 333

Tatematsu, K., Ohashi, S., Umemoto, T., et al. 2014, PASJ, 66, 16

Tatematsu, K., Liu, T., Ohashi, S., et al. 2017, ApJS, 228, 12

Tatematsu, K., Liu, T., Kim, G., et al. 2020, ApJ, 895, 119
Tobin, J. J., Stutz, A. M., Megeath, S. T., et al. 2015, ApJ, 798,128

Turner, B. E., \& Thaddeus, P. 1977, ApJ, 211, 755

Robitaille, T., Ginsburg, A., Beaumont, C., Leroy, A., \& Rosolowsky, E. 2016, Astrophysics Source Code Library, record ascl:1609.017

van Moorsel, G., Kemball, A., \& Greisen, E. 1996 in A. S. P. Conf. Ser. 101, Astronomical Data Analysis Software and Systems V, ed. G. H. Jacoby \& J. Barnes (San Franciso, CA: ASP), 37

Wolkovitch, D., Langer, W. D., Goldsmith, P. F., Heyer, M. 1997, ApJ, 477, 241

Womack, M., Ziurys, L. M., \& Sage, L. J. 1993, ApJ, 406, L29

Wright, E. L., Eisenhardt, P. R. M., Mainzer, A. K., et al. 2010, AJ, 140, 1868

Yamamoto, S., Saito, S., Kawaguchi, K., et al. 1990, ApJ, 361,318

Yi, H.-W., Lee, J.-E., Liu, T., et al. 2018, ApJS, 236, 51

Young, C. H., Shirley, Y. L., Evans, N. J. I., \& Rawlings, J. M. C. 2003, ApJS, 145, 111

Zhou, S., Wu, Y., Evans, N. J., II, Fuller, G. A., \& Myers, P. C. 1989, ApJ, 346, 168 


\section{ACKNOWLEDGMENTS}

K.T. and T.S. were supported by JSPS KAKENHI Grant Number 20H05645. J.H. thanks the National Natural Science Foundation of China under grant Nos. 11873086 and U1631237 and support by the Yunnan Province of China (No.2017HC018), and his work is sponsored in part by the Chinese Academy of Sciences (CAS), through a grant to the CAS South America Center for Astronomy (CASSACA) in Santiago, Chile.

\section{Facilities: No:45m}

Software: AIPS (van Moorsel et al. 1996), APLpy (Robitaille and Bressert 2012), Astropy (Astropy Collaboration et al. 2013, 2018), NOSTAR (Sawada et al. 2008), SAOImageDS9 (Joye \& Mandel 2003), spectral-cube (Robitaille et al. 2016)

\section{APPENDIX}

\section{A. MAPS}

Tables A1 and A2 summarize the field name, map-center coordinates, map size, distance, region, SCUBA-2 core name, core coordinates, name of associated YSO, and YSO coordinates as well as additional information. We list cores in the order employed in Kim et al. (2020) for consistency. Namely, we first list the Orion sources in the order of increasing Galactic longitude, and then the other sources in the order of increasing Right Ascension. Figure Set 19 (37 images), which is available in the online journal, shows the integrated-intensity contour maps of the 37 fields in the $\mathrm{N}_{2} \mathrm{H}^{+}, \mathrm{HC}_{3} \mathrm{~N}$, CCS-H, and CCS-L lines in the order of Tables 3 and A1. Note that Figures 1 to 7 are located in the main text. The velocity integration ranges, peak intensity, and rms noise level for the integrated intensity contour map are summarized in Table A3. The velocity range for $\mathrm{N}_{2} \mathrm{H}^{+}$is selected for the hyperfine group $J=1 \rightarrow 0 F_{1}=$ $2 \rightarrow 1$ containing the brightest hyperfine components.

As explained in $\S 3.4$, we fitted two-dimensional Gaussians to the spatial emission features of the Orion cores in the four lines. The coordinates of the emission feature centers and the deconvolved radii of the emission are listed in Tables A4 and A5. 


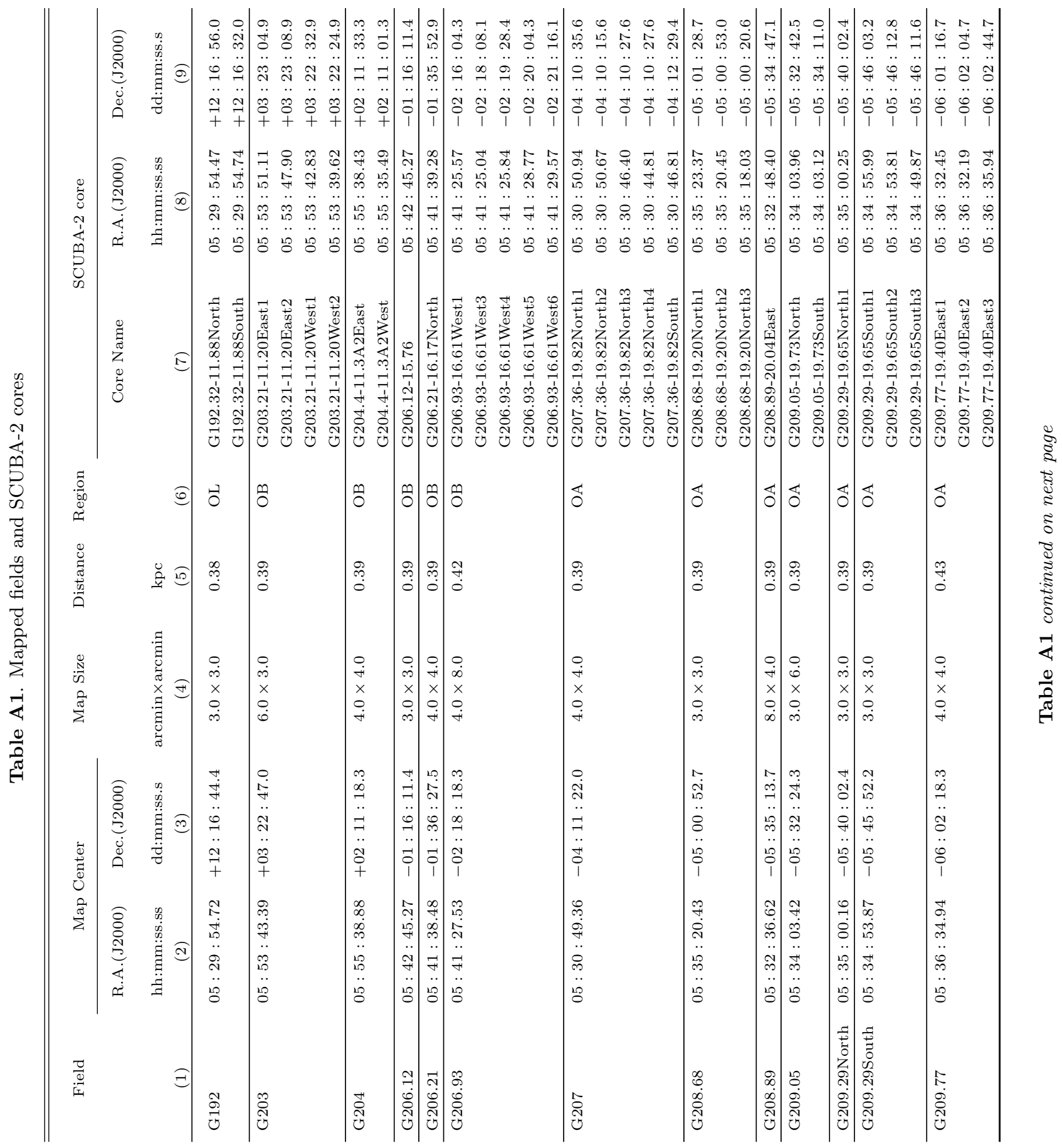




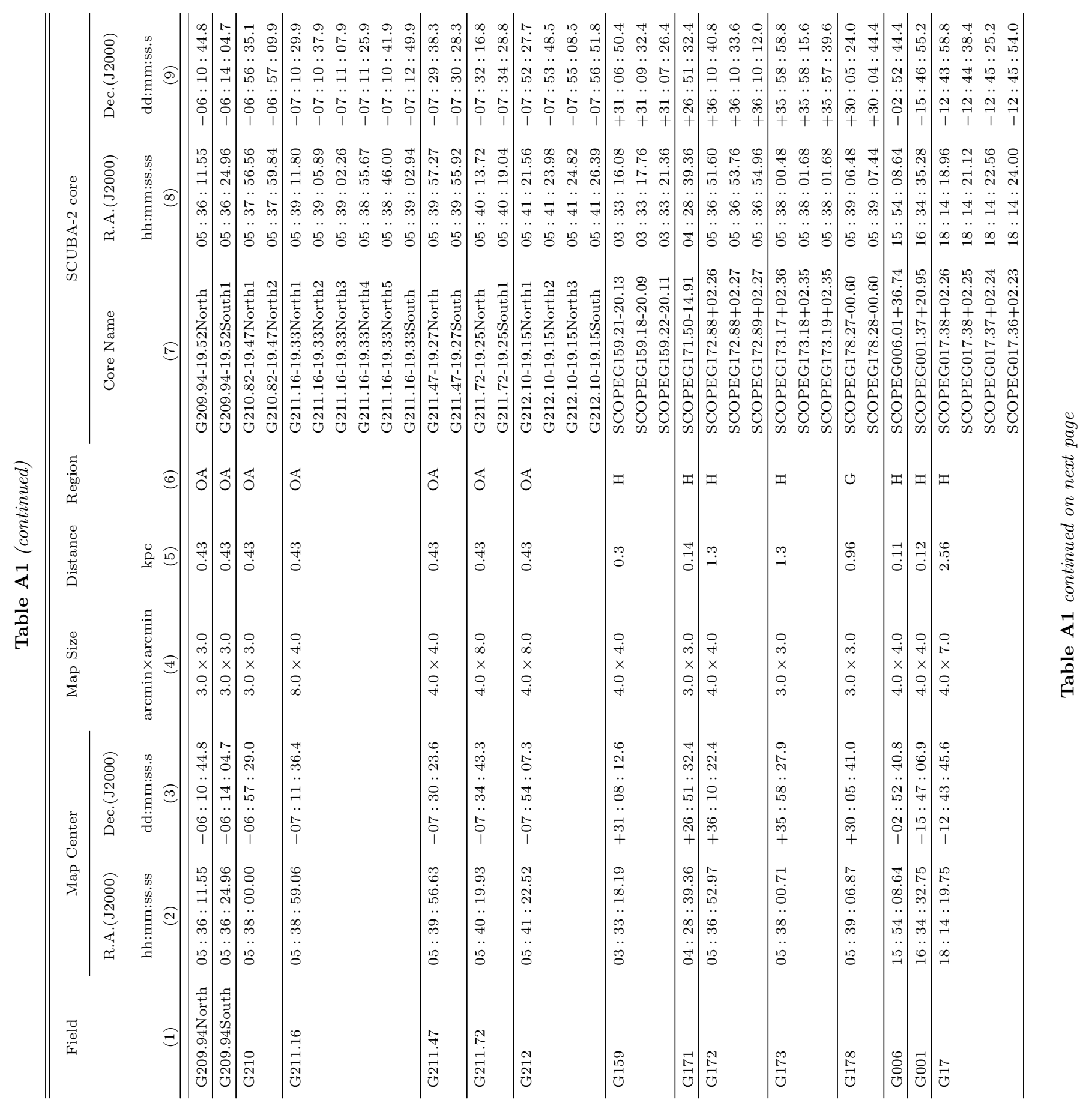




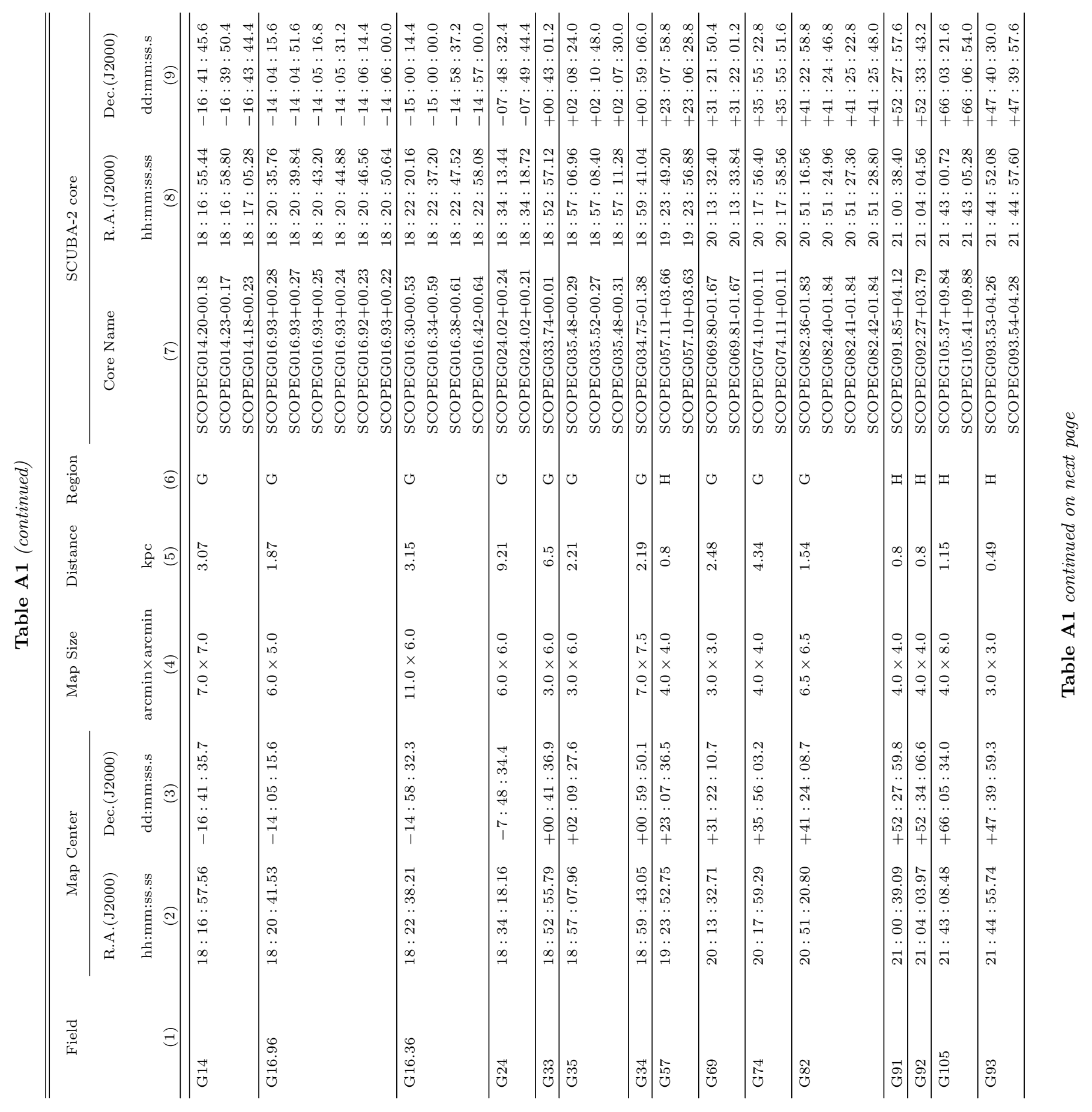




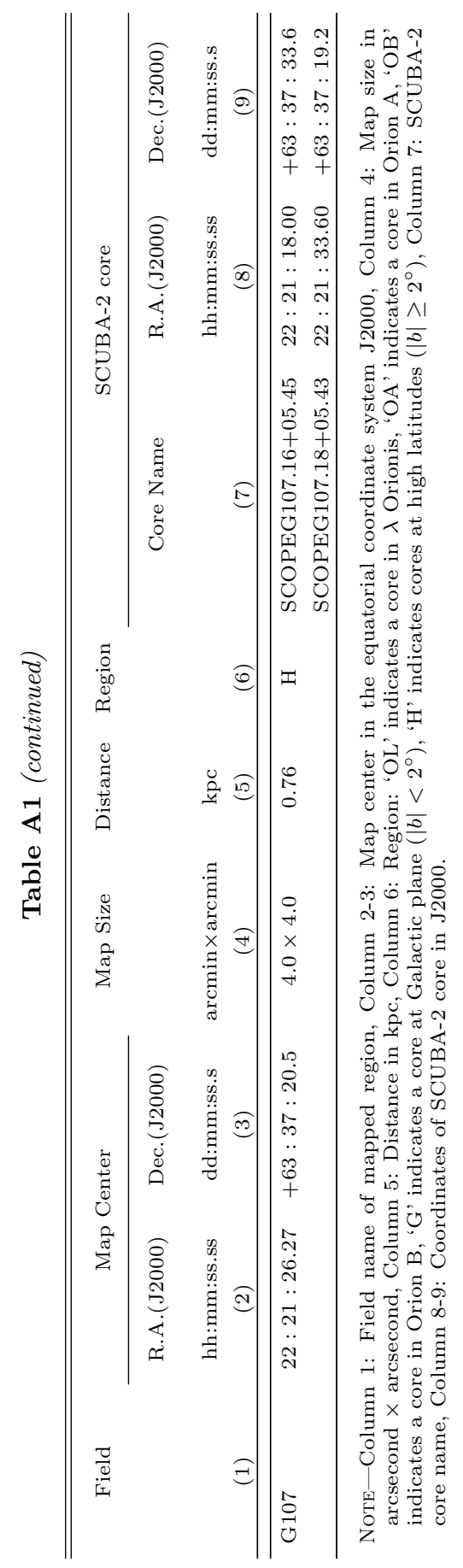


Table A2. Basic information of YSOs associated with SCUBA-2 cores

\begin{tabular}{|c|c|c|c|c|c|c|c|}
\hline SCUBA-2 core & Class & YSO name & $\begin{array}{c}\text { R.A.(J2000) } \\
\text { hh:mm:ss.ss } \\
(4)\end{array}$ & $\begin{array}{c}\text { Dec.(J2000) } \\
\text { dd:mm:ss.s } \\
(5) \\
\end{array}$ & $\begin{array}{c}T_{\mathrm{bol}} \\
\mathrm{K} \\
(6) \\
\end{array}$ & $\begin{array}{l}L_{\mathrm{bol}} \\
L_{\odot} \\
(7) \\
\end{array}$ & $\begin{array}{c}\theta_{\text {core-YSO }}-\mathrm{YSO} \\
\operatorname{arcsec} \\
(8) \\
\end{array}$ \\
\hline G192.32-11.88North & P0 & [LZK2016] G192N & $05: 29: 54.16$ & $+12: 16: 53.1$ & $\ldots$ & $\ldots$ & 5.4 \\
\hline G192.32-11.88South & $\mathrm{P}$ & [LZK2016] G192S & $05: 29: 54.35$ & $+12: 16: 29.7$ & $60 \pm 13$ & $0.1 \pm 0.1$ & 6.2 \\
\hline G203.21-11.20East 1 & $\mathrm{~S}$ & $\ldots$ & $\ldots$ & $\ldots$ & $\ldots$ & $\ldots$ & $\ldots$ \\
\hline G203.21-11.20East2 & $\mathrm{S}$ & $\ldots$ & $\ldots$ & $\ldots$ & $\ldots$ & $\ldots$ & $\ldots$ \\
\hline G203.21-11.20West1 & $\mathrm{P} 0$ & Herschel J055342.5+032236 & $05: 53: 42.51$ & $+03: 22: 35.7$ & $\ldots$ & $\ldots$ & 5.6 \\
\hline G203.21-11.20West2 & $\mathrm{P} 0$ & Herschel J055339.5+032225 & $05: 53: 39.55$ & $+03: 22: 25.4$ & $15 \pm 5$ & $0.5 \pm 0.3$ & 1.2 \\
\hline G204.4-11.3A2East & $\mathrm{P}$ & Herschel J055538.2+021135 & $05: 55: 38.23$ & $+02: 11: 35.2$ & $\ldots$ & $\ldots$ & 3.5 \\
\hline G204.4-11.3A2West & $\mathrm{P}$ & Herschel J055535.3+021103 & $05: 55: 35.36$ & $+02: 11: 03.2$ & $\ldots$ & $\ldots$ & 2.7 \\
\hline G206.12-15.76 & $\mathrm{P} 0$ & HOPS 400 & $05: 42: 45.23$ & $-01: 16: 14.2$ & $35 \pm 9$ & $3.0 \pm 1.4$ & 2.9 \\
\hline G206.21-16.17North & $\mathrm{S}$ & $\ldots$ & $\ldots$ & $\ldots$ & $\ldots$ & $\ldots$ & $\ldots$ \\
\hline G206.93-16.61West1 & PI & HOPS 300 & $05: 41: 24.21$ & $-02: 16: 06.5$ & $\ldots$ & $\ldots$ & 20.5 \\
\hline G206.93-16.61West3 & $\mathrm{P} 0$ & HOPS 399 & $05: 41: 24.94$ & $-02: 18: 08.5$ & $31 \pm 10$ & $6.3 \pm 3.0$ & 1.6 \\
\hline G206.93-16.61West 4 & $\mathrm{~S}$ & $\ldots$ & $\ldots$ & $\ldots$ & $\ldots$ & $\ldots$ & $\ldots$ \\
\hline G206.93-16.61West5 & $\mathrm{S}$ & $\ldots$ & $\ldots$ & $\ldots$ & $\ldots$ & $\ldots$ & $\ldots$ \\
\hline G206.93-16.61West6 & $\mathrm{P} 0$ & HOPS 398 & $05: 41: 29.40$ & $-02: 21: 17.1$ & $\ldots$ & $\ldots$ & 2.7 \\
\hline G207.36-19.82North1 & PI & VISION J05305129-0410322 & $05: 30: 51.29$ & $-04: 10: 32.2$ & $\ldots$ & $\cdots$ & 6.2 \\
\hline G207.36-19.82North2 & PI & VISION J05305129-0410322 & $05: 30: 51.29$ & $-04: 10: 32.2$ & $\ldots$ & $\ldots$ & 19.0 \\
\hline G207.36-19.82North3 & $\mathrm{S}$ & $\ldots$ & $\ldots$ & $\ldots$ & $\ldots$ & $\ldots$ & $\ldots$ \\
\hline G207.36-19.82North4 & $\mathrm{S}$ & . . & $\ldots$ & $\ldots$ & $\ldots$ & $\ldots$ & $\ldots$ \\
\hline G207.36-19.82South & $\mathrm{S}$ & $\ldots$ & $\ldots$ & $\ldots$ & $\ldots$ & $\ldots$ & $\ldots$ \\
\hline G208.68-19.20North1 & P0 & HOPS 87 & $05: 35: 23.47$ & $-05: 01: 28.7$ & $38 \pm 13$ & $36.7 \pm 14.5$ & 1.5 \\
\hline G208.68-19.20North2 & $\mathrm{PF}$ & HOPS 89 & $05: 35: 19.96$ & $-05: 01: 02.6$ & $112 \pm 10$ & $2.1 \pm 1.3$ & 12.1 \\
\hline G208.68-19.20North3 & $\mathrm{PF}$ & HOPS 92 & $05: 35: 18.32$ & $-05: 00: 33.0$ & $158 \pm 20$ & $22.0 \pm 8.7$ & 13.1 \\
\hline G208.89-20.04East & PI & WISE J053248.59-053451.2 & $05: 32: 48.60$ & $-05: 34: 51.3$ & $108 \pm 25$ & $2.2 \pm 1.0$ & 5.1 \\
\hline G209.05-19.73North & $\mathrm{S}$ & $\ldots$ & $\ldots$ & $\ldots$ & $\ldots$ & $\ldots$ & $\ldots$ \\
\hline G209.05-19.73South & $\mathrm{S}$ & $\cdots$ & $\cdots$ & $\ldots$ & $\cdots$ & $\cdots$ & $\cdots$ \\
\hline G209.29-19.65North1 & $\mathrm{S}$ & $\cdots$ & $\ldots$ & $\ldots$ & $\ldots$ & $\cdots$ & $\cdots$ \\
\hline G209.29-19.65South1 & $\mathrm{S}$ & $\cdots$ & $\ldots$ & $\ldots$ & $\cdots$ & $\cdots$ & $\cdots$ \\
\hline G209.29-19.65South2 & $\mathrm{S}$ & $\ldots$ & $\ldots$ & $\ldots$ & $\ldots$ & $\ldots$ & \\
\hline G209.29-19.65South2 & $\mathrm{S}$ & $\ldots$ & $\ldots$ & $\ldots$ & $\ldots$ & $\cdots$ & $\ldots$ \\
\hline G209.29-19.65South3 & PII & [MGM2012] 1221 & $05: 34: 49.08$ & $-05: 46: 04.8$ & $\ldots$ & $\ldots$ & 13.6 \\
\hline G209.77-19.40East1 & $\mathrm{PF}$ & HOPS 192 & $05: 36: 32.45$ & $-06: 01: 16.2$ & $\ldots$ & $\ldots$ & 0.5 \\
\hline G209.77-19.40East2 & $\mathrm{S}$ & $\ldots$ & $\ldots$ & $\ldots$ & $\ldots$ & $\cdots$ & $\ldots$ \\
\hline G209.77-19.40East3 & $\mathrm{S}$ & $\ldots$ & $\ldots$ & $\ldots$ & $\ldots$ & $\ldots$ & $\ldots$ \\
\hline G209.94-19.52North & PII & [MGM2012] 1025 & $05: 36: 09.66$ & $-06: 10: 30.6$ & $\ldots$ & $\cdots$ & 31.6 \\
\hline G209.94-19.52South1 & $\mathrm{S}$ & $\ldots$ & $\ldots$ & $\ldots$ & $\ldots$ & $\ldots$ & $\ldots$ \\
\hline G210.82-19.47North1 & PI & HOPS 157 & $05: 37: 56.57$ & $-06: 56: 39.2$ & $\ldots$ & $\ldots$ & 4.1 \\
\hline G210.82-19.47North2 & $\mathrm{S}$ & $\ldots$ & $\ldots$ & $\ldots$ & $\ldots$ & $\ldots$ & $\ldots$ \\
\hline G211.16-19.33North1 & $\mathrm{PF}$ & HOPS 129 & $05: 39: 11.85$ & $-07: 10: 35.0$ & $\ldots$ & $\ldots$ & 5.2 \\
\hline G211.16-19.33North2 & PI & HOPS 133 & $05: 39: 05.83$ & $-07: 10: 39.4$ & $70 \pm 20$ & $3.7 \pm 1.4$ & 1.7 \\
\hline G211.16-19.33North3 & $\mathrm{S}$ & $\ldots$ & $\ldots$ & $\ldots$ & $\ldots$ & $\ldots$ & $\ldots$ \\
\hline G211.16-19.33North4 & $\mathrm{P}$ & Herschel J053854.1-071123 & $05: 38: 54.13$ & $-07: 11: 22.8$ & $\ldots$ & $\ldots$ & 23.1 \\
\hline G211.16-19.33North5 & PI & HOPS 135 & $05: 38: 45.31$ & $-07: 10: 55.9$ & $112 \pm 16$ & $1.3 \pm 0.5$ & 17.3 \\
\hline G211.16-19.33South & PI & HOPS 130 & $05: 39: 02.96$ & $-07: 12: 52.3$ & $\ldots$ & $\ldots$ & 2.4 \\
\hline G211.47-19.27North & $\mathrm{P} 0$ & HOPS 290 & $05: 39: 57.41$ & $-07: 29: 33.4$ & $48 \pm 10$ & $4.0 \pm 1.7$ & 5.3 \\
\hline G211.47-19.27South & P0 & HOPS 288 & $05: 39: 55.94$ & $-07: 30: 28.0$ & $49 \pm 21$ & $180.0 \pm 70.0$ & 0.5 \\
\hline G211.72-19.25North & PII & WISE J054013.78-073216.0 & $05: 40: 13.79$ & $-07: 32: 16.1$ & $\ldots$ & $\ldots$ & 1.2 \\
\hline G211.72-19.25South1 & $\mathrm{S}$ & $\ldots$ & $\ldots$ & $\ldots$ & $\ldots$ & $\ldots$ & $\ldots$ \\
\hline G212.10-19.15North1 & $\mathrm{P}$ & Herschel J054120.5-075237 & $05: 41: 20.55$ & $-07: 52: 36.6$ & $\ldots$ & $\ldots$ & 17.5 \\
\hline
\end{tabular}

Table A2 continued on next page 
Table A2 (continued)

\begin{tabular}{|c|c|c|c|c|c|c|c|}
\hline SCUBA-2 core & Class & YSO name & $\begin{array}{c}\text { R.A.(J2000) } \\
\text { hh:mm:ss.ss } \\
(4)\end{array}$ & $\begin{array}{c}\text { Dec.(J2000) } \\
\text { dd:mm:ss.s } \\
(5)\end{array}$ & $\begin{array}{c}T_{\mathrm{bol}} \\
\mathrm{K} \\
(6) \\
\end{array}$ & $\begin{array}{c}L_{\text {bol }} \\
L_{\odot} \\
(7) \\
\end{array}$ & $\begin{array}{c}\theta_{\text {core-YSO }} \\
\text { arcsec } \\
(8) \\
\end{array}$ \\
\hline G212.10-19.15North2 & PI & HOPS 263 & $05: 41: 23.68$ & $-07: 53: 46.8$ & $114 \pm 10$ & $1.1 \pm 0.5$ & 4.7 \\
\hline G212.10-19.15North3 & PI & HOPS 254 & $05: 41: 24.52$ & $-07: 55: 07.3$ & $\ldots$ & $\ldots$ & 4.7 \\
\hline G212.10-19.15South & $\mathrm{P} 0$ & HOPS 247 & $05: 41: 26.22$ & $-07: 56: 51.6$ & $43 \pm 12$ & $3.2 \pm 1.2$ & 2.5 \\
\hline SCOPEG159.21-20.13 & $\mathrm{P} 0$ & [EES2009] Per-emb 10 & $03: 33: 16.45$ & $+31: 06: 52.5$ & $\ldots$ & $\ldots$ & 5.2 \\
\hline SCOPEG159.18-20.09 & P0 & SSTc2d J033317.8+310931 & $03: 33: 17.85$ & $+31: 09: 31.9$ & $\cdots$ & $\cdots$ & 1.3 \\
\hline SCOPEG159.22-20.11 & P0 & [SDA2014] West41 & $03: 33: 21.30$ & $+31: 07: 27.0$ & $\ldots$ & $\ldots$ & 1.0 \\
\hline SCOPEG171.50-14.91 & PI & NAME LDN $1521 \mathrm{~F}$ IRS & $04: 28: 38.95$ & $+26: 51: 35.1$ & $\ldots$ & $\cdots$ & 6.1 \\
\hline SCOPEG172.88+02.26 & $\mathrm{P}$ & WISE J053651.31+361037.1 & $05: 36: 51.31$ & $+36: 10: 37.2$ & $\ldots$ & $\cdots$ & 5.1 \\
\hline SCOPEG $172.88+02.27$ & $\mathrm{P}$ & WISE J053653.75+361033.5 & $05: 36: 53.76$ & $+36: 10: 33.6$ & $\ldots$ & $\ldots$ & 0.0 \\
\hline SCOPEG172.89+02.27 & $\mathrm{P}$ & WISE J053654.91+361008.2 & $05: 36: 54.91$ & $+36: 10: 08.3$ & $\cdots$ & $\cdots$ & 3.7 \\
\hline SCOPEG173.17+02.36 & PI & WISE J053800.11+355903.7 & $05: 38: 00.12$ & $+35: 59: 03.7$ & $\ldots$ & $\ldots$ & 6.6 \\
\hline SCOPEG173.18+02.35 & $\mathrm{P}$ & Herschel J053801.5+355817 & $05: 38: 01.54$ & $+35: 58: 17.2$ & $\cdots$ & $\cdots$ & 2.3 \\
\hline SCOPEG173.19+02.35 & $\mathrm{P}$ & Herschel J053801.3+355734 & $05: 38: 01.32$ & $+35: 57: 33.5$ & $\ldots$ & $\cdots$ & 7.5 \\
\hline SCOPEG178.27-00.60 & $\mathrm{P}$ & Herschel J053906.4+300524 & $05: 39: 06.47$ & $+30: 05: 24.4$ & $\ldots$ & $\ldots$ & 0.4 \\
\hline SCOPEG178.28-00.60 & $\mathrm{P}$ & Herschel J053907.3+300452 & $05: 39: 07.31$ & $+30: 04: 51.9$ & $\ldots$ & $\cdots$ & 7.7 \\
\hline SCOPEG006.01+36.74 & $\mathrm{S}$ & $\cdots$ & $\ldots$ & $\ldots$ & $\cdots$ & $\cdots$ & $\cdots$ \\
\hline SCOPEG001.37+20.95 & $\mathrm{S}$ & $\ldots$ & $\ldots$ & $\ldots$ & $\ldots$ & $\cdots$ & $\cdots$ \\
\hline SCOPEG017.38+02.26 & $\mathrm{P}$ & WISE J181418.81-124358.7 & $18: 14: 18.82$ & $-12: 43: 58.7$ & $\ldots$ & $\cdots$ & 2.1 \\
\hline SCOPEG017.38+02.25 & PI & MSX6C G017.3765+02.2512 & $18: 14: 21.10$ & $-12: 44: 33.0$ & $\ldots$ & $\ldots$ & 5.4 \\
\hline SCOPEG017.37+02.24 & $\mathrm{P}$ & WISE J181422.18-124521.2 & $18: 14: 22.18$ & $-12: 45: 21.2$ & $\ldots$ & $\ldots$ & 6.8 \\
\hline SCOPEG017.36+02.23 & $\mathrm{P}$ & WISE J181424.00-124548.6 & $18: 14: 24.00$ & $-12: 45: 48.6$ & $\cdots$ & $\cdots$ & 5.4 \\
\hline SCOPEG014.20-00.18 & $\mathrm{P}$ & WISE J181656.04-164141.5 & $18: 16: 56.04$ & $-16: 41: 41.6$ & $\ldots$ & $\cdots$ & 9.6 \\
\hline SCOPEG014.23-00.17 & $\mathrm{S}$ & $\ldots$ & $\ldots$ & $\ldots$ & $\ldots$ & $\ldots$ & $\ldots$ \\
\hline SCOPEG014.18-00.23 & $\mathrm{P}$ & Herschel G014.1897-0.2273 & $18: 17: 05.61$ & $-16: 43: 28.3$ & $\cdots$ & $\cdots$ & 16.8 \\
\hline SCOPEG016.93+00.28 & $\mathrm{P}$ & MSX6C G016.9261+00.2854 & $18: 20: 35.50$ & $-14: 04: 16.0$ & $\ldots$ & $\cdots$ & 3.8 \\
\hline SCOPEG016.93+00.27 & $\mathrm{P}$ & Herschel G016.9253+0.2662 & $18: 20: 39.57$ & $-14: 04: 51.2$ & $\ldots$ & $\cdots$ & 4.0 \\
\hline SCOPEG016.93+00.25 & $\mathrm{S}$ & $\ldots$ & $\ldots$ & $\ldots$ & $\ldots$ & $\cdots$ & $\cdots$ \\
\hline SCOPEG016.93+00.25 & $\mathrm{S}$ & . . & $\cdots$ & $\cdots$ & $\cdots$ & $\cdots$ & $\cdots$ \\
\hline SCOPEG016.93+00.24 & $\mathrm{S}$ & . . & $\cdots$ & $\cdots$ & $\cdots$ & $\cdots$ & $\cdots$ \\
\hline SCOPEG016.93+00.24 & $\mathrm{S}$ & . . & $\ldots$ & $\ldots$ & $\ldots$ & $\ldots$ & $\ldots$ \\
\hline SCOPEG016.92+00.23 & $\mathrm{S}$ & $\ldots$ & $\ldots$ & $\ldots$ & $\cdots$ & $\cdots$ & $\cdots$ \\
\hline SCOPEG016.93+00.22 & $\mathrm{P}$ & Herschel G016.9288+0.2180 & $18: 20: 50.48$ & $-14: 06: 01.8$ & $\cdots$ & $\cdots$ & 2.9 \\
\hline SCOPEG016.30-00.53 & $\mathrm{S}$ & $\ldots$ & $\ldots$ & $\ldots$ & $\ldots$ & $\ldots$ & $\ldots$ \\
\hline SCOPEG016.34-00.59 & PI & WISE J182236.21-145956.5 & $18: 22: 36.21$ & $-14: 59: 56.6$ & $\ldots$ & $\cdots$ & 14.7 \\
\hline SCOPEG016.38-00.61 & $\mathrm{P}$ & Herschel J182247.7-145848 & $18: 22: 47.71$ & $-14: 58: 47.5$ & $\cdots$ & $\cdots$ & 10.7 \\
\hline SCOPEG016.42-00.64 & $\mathrm{S}$ & . . & $\ldots$ & $\ldots$ & $\ldots$ & $\cdots$ & $\ldots$ \\
\hline SCOPEG016.42-00.64 & $\mathrm{S}$ & . . & $\cdots$ & $\cdots$ & $\cdots$ & $\cdots$ & $\cdots$ \\
\hline SCOPEG024.02+00.24 & $\mathrm{S}$ & . . & $\cdots$ & $\cdots$ & $\cdots$ & $\cdots$ & $\cdots$ \\
\hline SCOPEG024.02+00.21 & $\mathrm{S}$ & $\cdots$ & $\ldots$ & $\ldots$ & $\cdots$ & $\cdots$ & $\ldots$ \\
\hline SCOPEG033.74-00.01 & $\mathrm{P}$ & Herschel J185256.6+004316 & $18: 52: 56.68$ & $+00: 43: 15.5$ & $\cdots$ & $\cdots$ & 15.8 \\
\hline SCOPEG035.48-00.29 & $\mathrm{P}$ & SSTGLMC G035.4858-00.2876 & $18: 57: 07.40$ & $+02: 08: 29.2$ & $\cdots$ & $\cdots$ & 8.4 \\
\hline SCOPEG035.52-00.27 & $\mathrm{P}$ & Herschel G035.5235-0.2728 & $18: 57: 08.34$ & $+02: 10: 54.5$ & $\cdots$ & $\cdots$ & 6.5 \\
\hline SCOPEG035.48-00.31 & $\mathrm{P}$ & MIREX G035.4823-00.3086 & $18: 57: 11.50$ & $+02: 07: 43.6$ & $\cdots$ & $\cdots$ & 14.0 \\
\hline SCOPEG034.75-01.38 & $\mathrm{P}$ & Herschel J185941.2+005908 & $18: 59: 41.23$ & $+00: 59: 08.1$ & $\cdots$ & $\cdots$ & 3.5 \\
\hline SCOPEG057.11+03.66 & $\mathrm{S}$ & $\ldots$ & $\ldots$ & $\ldots$ & $\cdots$ & $\cdots$ & $\cdots$ \\
\hline SCOPEG057.10+03.63 & $\mathrm{P}$ & WISE J192356.78+230633.0 & $19: 23: 56.78$ & $+23: 06: 33.1$ & $\ldots$ & $\ldots$ & 4.5 \\
\hline SCOPEG069.80-01.67 & PI & [MJR2015] 3117 & $20: 13: 32.55$ & $+31: 22: 00.4$ & $\ldots$ & $\ldots 10.2$ & \\
\hline SCOPEG069.81-01.67 & $\mathrm{P}$ & WISE J201333.64+312206.3 & $20: 13: 33.64$ & $+31: 22: 06.3$ & $\cdots$ & $\ldots$ & 5.7 \\
\hline SCOPEG074.10+00.11 & $\mathrm{P}$ & Herschel J201756.3+355525 & $20: 17: 56.30$ & $+35: 55: 25.3$ & $\cdots$ & $\cdots$ & 2.8 \\
\hline SCOPEG074.11+00.11 & $\mathrm{P}$ & WISE J201758.56+355552.3 & $20: 17: 58.57$ & $+35: 55: 52.4$ & $\cdots$ & $\cdots$ & 0.8 \\
\hline SCOPEG082.36-01.83 & $\mathrm{S}$ & $\ldots$ & $\ldots$ & $\ldots$ & $\cdots$ & $\cdots$ & $\cdots$ \\
\hline SCOPEG082.40-01.84 & $\mathrm{P}$ & [MJR2015] 3335 & $20: 51: 24.82$ & $+41: 24: 49.5$ & $\ldots$ & $\ldots$ & 3.2 \\
\hline
\end{tabular}

Table A2 continued on next page 
Table A2 (continued)

\begin{tabular}{|c|c|c|c|c|c|c|c|}
\hline SCUBA-2 core & Class & YSO name & $\begin{array}{c}\text { R.A.(J2000) } \\
\text { hh:mm:ss.ss } \\
(4)\end{array}$ & $\begin{array}{c}\text { Dec.(J2000) } \\
\text { dd:mm:ss.s } \\
(5)\end{array}$ & $\begin{array}{c}T_{\text {bol }} \\
\mathrm{K} \\
(6) \\
\end{array}$ & $\begin{array}{c}L_{\text {bol }} \\
L_{\odot} \\
(7)\end{array}$ & $\begin{array}{c}\theta_{\text {core }-\mathrm{YSO}} \\
\operatorname{arcsec} \\
(8)\end{array}$ \\
\hline SCOPEG082.41-01.84 & $\mathrm{S}$ & . . & $\cdots$ & $\cdots$ & $\cdots$ & $\cdots$ & $\cdots$ \\
\hline SCOPEG082.42-01.84 & $\mathrm{S}$ & . . & $\ldots$ & $\ldots$ & $\cdots$ & $\cdots$ & $\cdots$ \\
\hline SCOPEG091.85+04.12 & $\mathrm{P}$ & WISE J210038.77+522757.5 & $21: 00: 38.76$ & $+52: 27: 57.5$ & $\cdots$ & $\cdots$ & 3.3 \\
\hline SCOPEG092.27+03.79 & PI & [MJR2015] 3554 & $21: 04: 04.50$ & $+52: 33: 47.2$ & $\ldots$ & 4.0 & \\
\hline SCOPEG105.37+09.84 & PI & [SS2009] NGC 7129-S3-U419 & $21: 43: 01.78$ & $+66: 03: 24.4$ & $\cdots$ & $\cdots$ & 7.0 \\
\hline SCOPEG105.41+09.88 & PIII & 2MASS J21430502+6606533 & $21: 43: 04.98$ & $+66: 06: 53.3$ & $\ldots$ & $\ldots$ & 2.0 \\
\hline SCOPEG093.53-04.26 & $\mathrm{P}$ & HHL 73 IRS 1 & $21: 44: 52.01$ & $+47: 40: 30.8$ & $\ldots$ & $\ldots$ & 1.1 \\
\hline SCOPEG093.54-04.28 & $\mathrm{S}$ & . . & $\ldots$ & $\ldots$ & $\cdots$ & $\cdots$ & $\ldots$ \\
\hline SCOPEG107.16+05.45 & PII & [MJR2015] 3797 & $22: 21: 20.15$ & $+63: 37: 37.8$ & $\ldots$ & $\ldots$ & 15.0 \\
\hline SCOPEG107.18+05.43 & $\mathrm{P}$ & [HLB98] Onsala 164 & $22: 21: 33.20$ & $+63: 37: 22.0$ & $\ldots$ & $\ldots$ & 3.9 \\
\hline
\end{tabular}

Note-Column 1: SCUBA-2 core name, Column 2: Core classification: ' $\mathrm{S}$ ' indicates a starless core, 'P' indicates a protostellar core, 'P0' indicates a core including class 0 protostar, 'PI' indicates a core including a class I protostar, 'PF' indicates a core including a flat-spectrum protostar, 'PII' indicates a core including a class II protostar, Column 3: name of young stellar object (YSO), Column 4-5: Coordinate of YSO in Equatorial system (J2000), Column 6: Bolometric temperature, Column 7: Separation between SCUBA-2 core and YSO in arcseconds. 


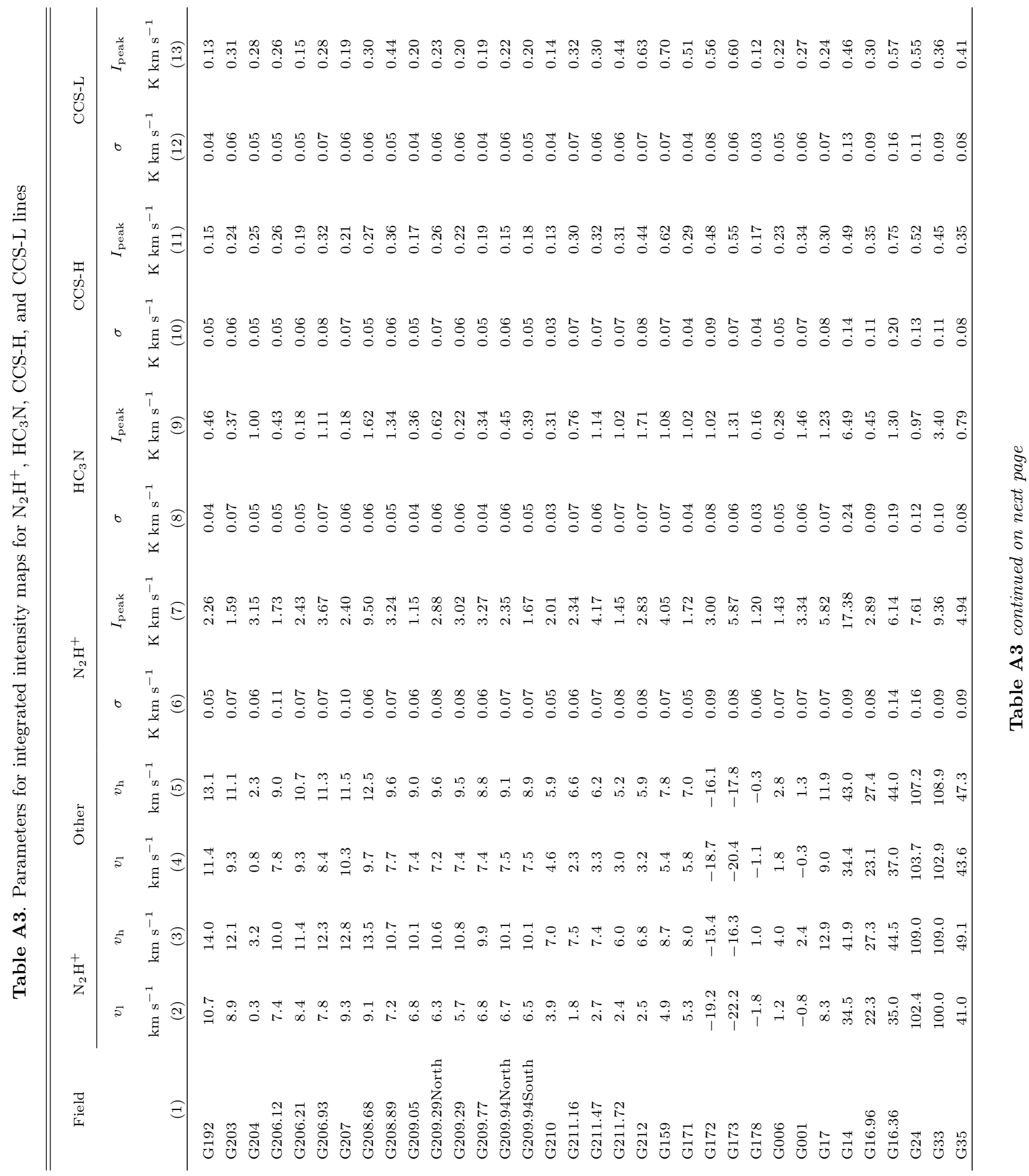




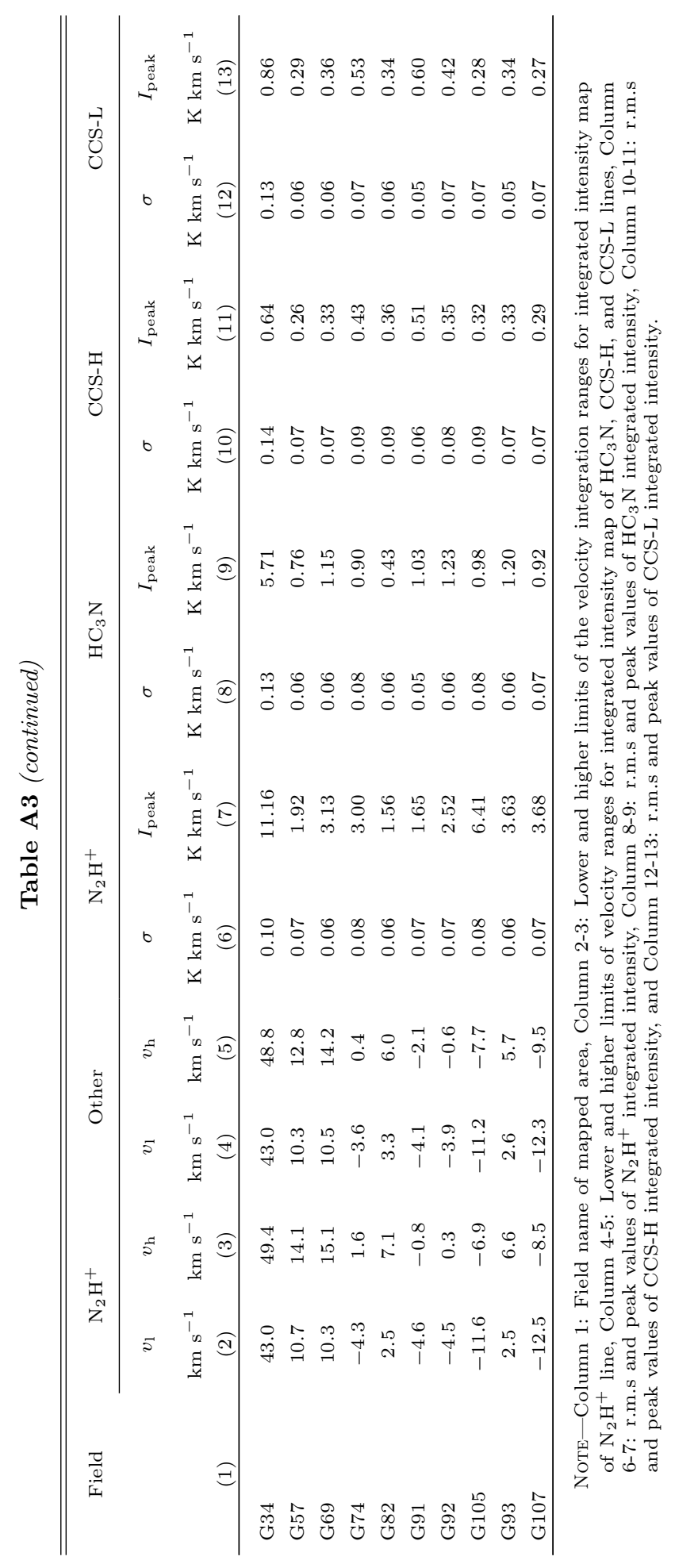




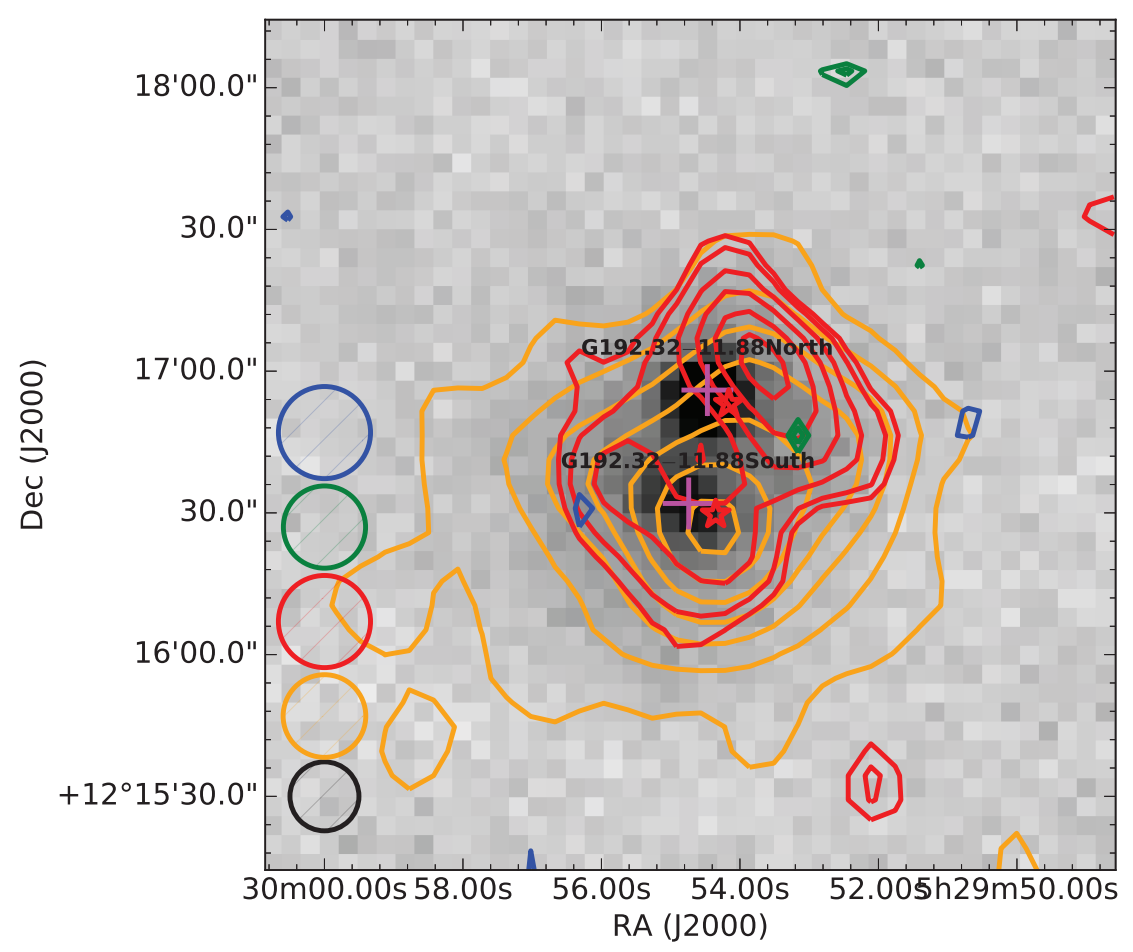

Figure 19. Same as Figure 1 but for the specified field

Fig. Set 19. $\mathrm{N}_{2} \mathrm{H}^{+}, \mathrm{HC}_{3} \mathrm{~N}, \mathrm{CCS}-\mathrm{H}$, and CCS-L maps overlaid on the JCMT SCUBA-2 map for the Appendix. 


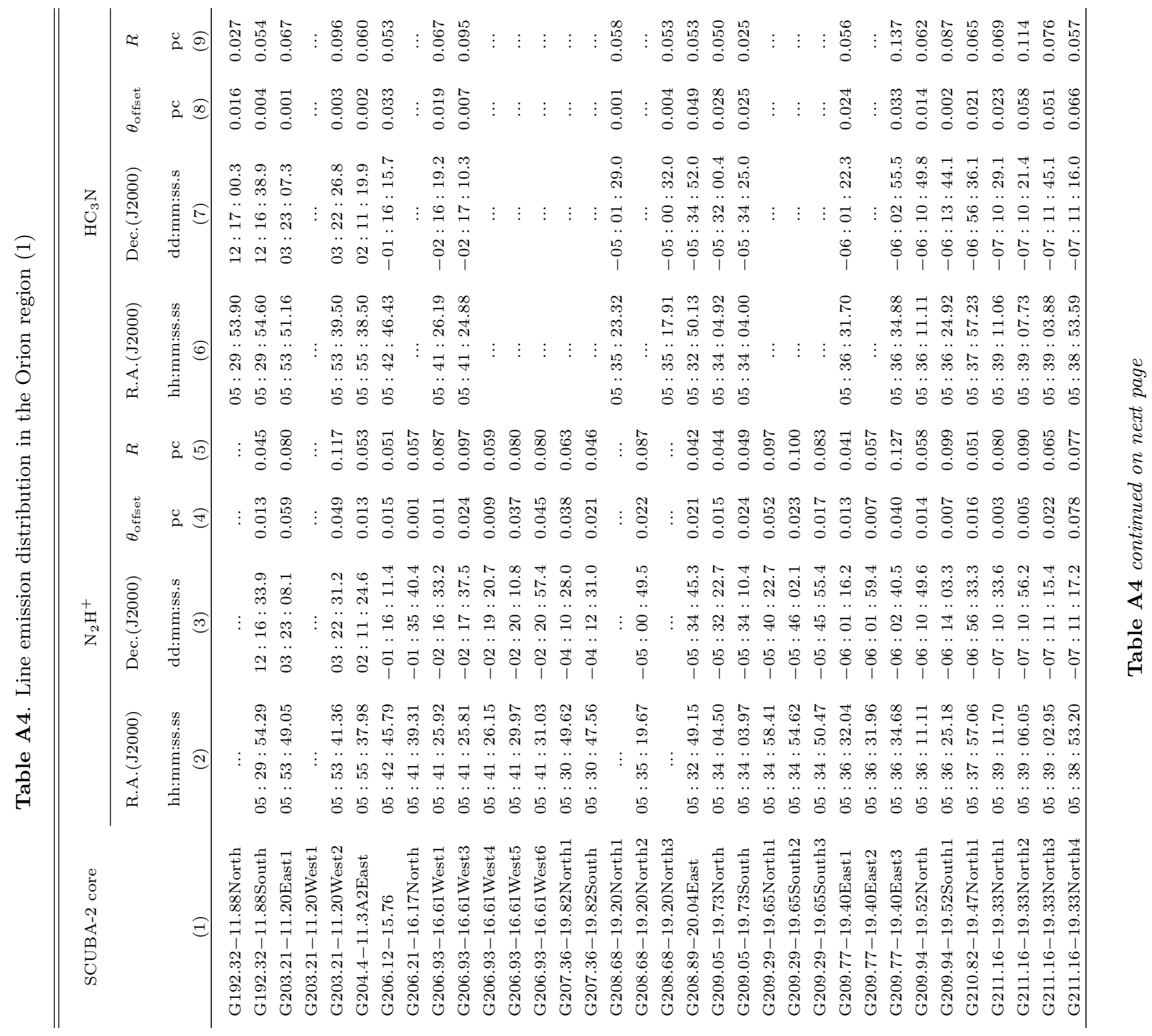




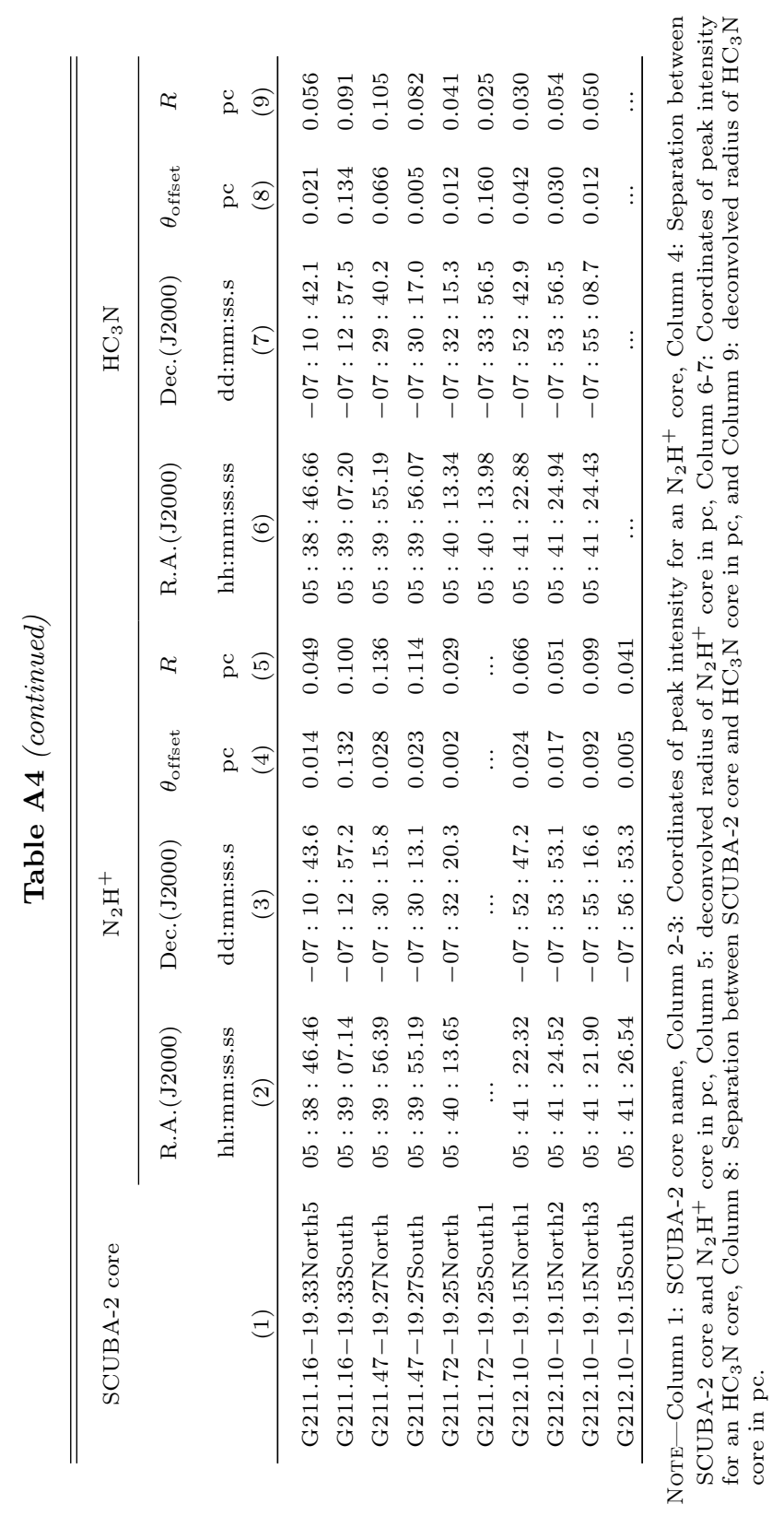




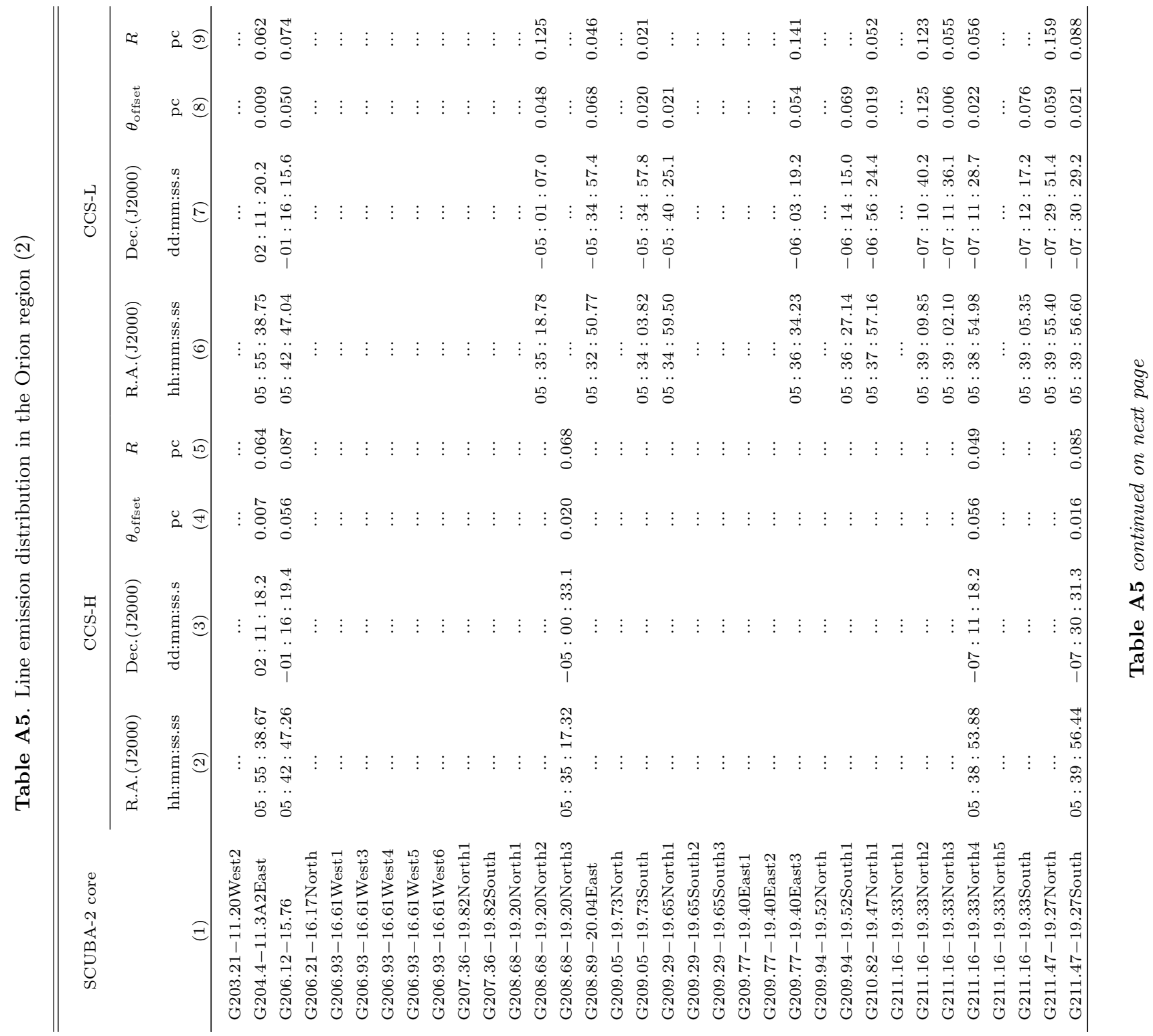




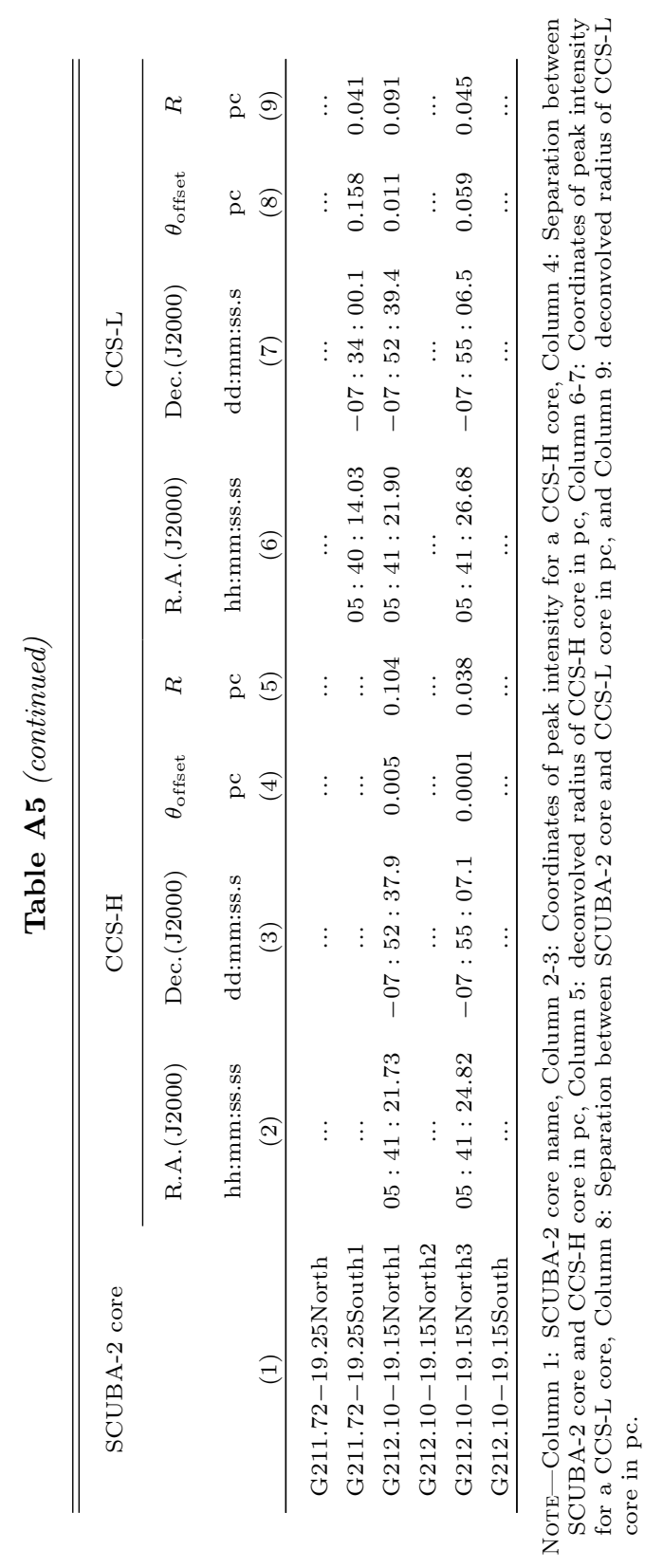

